\title{
Heisenberg algebra, wedges and crystals
}

\author{
Thomas Gerber ${ }^{1}$
}

Received: 6 March 2017 / Accepted: 8 March 2018 / Published online: 19 March 2018

C Springer Science+Business Media, LLC, part of Springer Nature 2018

\begin{abstract}
We explain how the action of the Heisenberg algebra on the space of $q$ deformed wedges yields the Heisenberg crystal structure on charged multipartitions, by using the Boson-Fermion correspondence and looking at the action of the Schur functions at $q=0$. In addition, we give the explicit formula for computing this crystal in full generality.
\end{abstract}

Keywords Fock space - Categorification - Quantum groups · Heisenberg algebra · Crystals $\cdot$ Symmetric functions $\cdot$ Combinatorics

\section{Introduction}

Categorification of representations of affine quantum groups has proved to be an important tool for understanding many classic objects arising from modular group representation theory, among which Hecke algebras and rational Cherednik algebras of cyclotomic type, and finite classical groups. More precisely, the study of crystals and canonical bases of the level $\ell$ Fock space representations $\mathcal{F}_{\mathrm{s}}$ of $\mathcal{U}_{q}^{\prime}\left(\widehat{\mathfrak{s l}_{e}}\right)$ gives answers to several classical problems in combinatorial terms. In particular, we know that the $\mathcal{U}_{q}^{\prime}\left(\widehat{\mathfrak{s l}_{e}}\right)$-crystal graph of $\mathcal{F}_{\mathbf{s}}$ can be categorified in the following ways:

- by the parabolic branching rule for modular cyclotomic Hecke algebras [1], when restricting to the connected component containing the empty $\ell$-partition,

- by Bezrukavnikov and Etingof's parabolic branching rule for cyclotomic rational Cherednik algebras [23],

\footnotetext{
Thomas Gerber

gerber@math.rwth-aachen.de

1 Lehrstuhl D für Mathematik, RWTH Aachen University, 52062 Aachen, Germany
} 
- by the weak Harish-Chandra modular branching rule on unipotent representations for finite unitary groups $[3,10]$, for $\ell=2$ and $\mathbf{s}$ varying.

In each case, the branching rule depends on some parameters that are explicitly determined by the parameters $e$ and $\mathbf{s}$ of the Fock space.

Recently, there has been some important developments when Shan and Vasserot [24] categorified the action of the Heisenberg algebra on a certain direct sum of Fock spaces, in order to prove a conjecture by Etingof [5]. Losev gave in [19] a formulation of Shan and Vasserot's results in terms of crystals, as well as an explicit formula for computing it in the asymptotic case (see Definition 3.5). Independently and using different methods, the author defined a notion of Heisenberg crystal for higher level Fock spaces [9], that turns out to coincide with Losev's crystal. An explicit formula was also given in another particular case, using level-rank duality. Like the $\mathcal{U}_{q}^{\prime}\left(\widehat{\mathfrak{s l}_{e}}\right)$-crystal, the Heisenberg crystal gives some information at the categorical level. In particular, it yields a characterisation of

- the finite-dimensional irreducible modules in the cyclotomic Cherednik category $\mathcal{O}$ by [24] and [9],

- the usual cuspidal irreducible unipotent modular representations of finite unitary groups [4].

This paper solves two remaining problems about the Heisenberg crystal. Firstly, even though it originally arises from the study of cyclotomic rational Cherednik algebras (it is determined by combinatorial versions of certain adjoint functors defined on the bounded derived category $\mathcal{O}$ ), the Heisenberg crystal has an intrinsic existence as shown in [9]. Therefore, it is natural to ask for an algebraic construction of the Heisenberg crystal which would be independent of any categorical interpretation. This is achieved via the Boson-Fermion correspondence and the use of the Schur functions, acting on Uglov's canonical basis of $\mathcal{F}_{\mathbf{s}}$. This gives a new realisation of the Heisenberg crystal, analogous to Kashiwara's crystals for quantum group. Secondly, we give an explicit formula for computing the Heisenberg crystal in full generality. This generalises and completes the particular results of [19] and [9]. This is done in the spirit of [6] where formulas for the $\mathcal{U}_{q}^{\prime}\left(\widehat{\mathfrak{s l}}_{e}\right)$-crystal were explicited.

The present paper has the following structure. In Sect. 2, we start by introducing in detail several combinatorial objects indexing the basis of the wedge space (namely charged multipartitions, abaci and wedges) and the different ways to identify them. Then, we quickly recall some essential facts about the $\mathcal{U}_{q}^{\prime}\left(\widehat{\mathfrak{s l}_{e}}\right)$-structure of the Fock spaces $\mathcal{F}_{\mathbf{s}}$. Section 3 focuses on the Heisenberg action on the wedge space $\mathcal{F}_{s}$, seen as a certain direct sum of level $\ell$ Fock spaces $\mathcal{F}_{\mathbf{s}}$. In particular, we recall the definition of the Heisenberg crystal given in [9]. Then, we give in Sect. 4 a solution to the first problem mentioned above. More precisely, we recall the quantum Boson-Fermion correspondence and fundamental facts about symmetric functions. Inspired by Shan and Vasserot [24] and Leclerc and Thibon [18], we study the action of the Schur functions on the wedge space and use a result of Iijima [11] to construct the Heisenberg crystal as a version of this action at $q=0$ (Theorem 4.4), resembling Kashiwara's philosophy of crystal and canonical bases. Most importantly, by doing so, we bypass entirely Shan and Vasserot's original categorical construction. Section 5 is devoted to the explicit computation of the Heisenberg crystal. We introduce level $\ell$ vertical 
$e$-strips, as well as the notion of good vertical $e$-strips by defining an appropriate order. The action of the Heisenberg crystal operators is then given in terms of adding good level $\ell$ vertical $e$-strips (Theorem 5.11), which is reminiscent of the explicit formula for the Kashiwara crystal operators first given in [14] (see also [6]). We relate this result to other combinatorial procedures in the literature answering in particular a question of Tingley [26]. In addition, we give several examples of explicit computations. Finally, we recall some useful facts about level-rank duality in "Appendix", enabling the definition of the Heisenberg crystal.

\section{Higher level Fock spaces}

\subsection{Charged multipartitions and wedges}

\subsubsection{Charged multipartitions}

Fix once and for all elements $e, \ell \in \mathbb{Z}_{\geq 2}$ and $s \in \mathbb{Z}$. An $\ell$-partition (or simply multipartition) is an $\ell$-tuple of partitions $\lambda=\left(\lambda^{1}, \ldots, \lambda^{\ell}\right)$. These will be represented using Young diagrams. Denote $\Pi_{\ell}$ the set of $\ell$-partitions and $\Pi=\Pi_{1}$ the set of partitions. Partitions will sometimes be denoted multiplicatively for convenience, e.g. $(2,1,1)=\left(2.1^{2}\right)=\square$.

An $\ell$-charge (or simply (multi)charge) is an $\ell$-tuple of integers $\mathbf{s}=\left(s_{1}, \ldots, s_{\ell}\right)$. We write $|\mathbf{s}|=\sum_{j=1}^{\ell} s_{j}$. We call charged $\ell$-partition the data consisting of an $\ell$-partition $\lambda$ and an $\ell$-charge $\mathbf{s}$, and denote it by $|\lambda, \mathbf{s}\rangle$.

For a box $\gamma=(a, b, j)$ in the Young diagram of $\lambda$ (where $a \in \mathbb{Z}$ is the row index of the box, $b \in \mathbb{Z}$ the column index and $j \in\{1, \ldots, \ell\}$ the coordinate), let $\mathfrak{c}(\gamma)=b-a+s_{j}$, the content of $\gamma$. We will represent $|\lambda, \mathbf{s}\rangle$ by filling the boxes of the Young diagram of $\lambda$ with their contents.

Example 2.1 Take $\ell=2, \mathbf{s}=(-1,2)$ and $\lambda=\left(2.1,1^{2}\right)$. We have $s=-1+2=1$ and

$$
|\lambda, \mathbf{s}\rangle=\left(\begin{array}{c}
\frac{-10}{-2}, \\
\frac{2}{1}
\end{array}\right)
$$

In the following, we will only consider multicharges $\mathbf{s}$ verifying $|\mathbf{s}|:=s_{1}+\cdots+s_{\ell}=$ $s$.

For a partition $\lambda$, let $\lambda^{\prime}$ denote its conjugate, that is the partition obtained by swapping rows and columns in the Young diagram of $\lambda$. We extend this to charged multipartitions by setting $|\lambda, \mathbf{s}\rangle^{\prime}=\left|\lambda^{\prime}, \mathbf{s}^{\prime}\right\rangle$ where $\lambda^{\prime}=\left(\left(\lambda^{\ell}\right)^{\prime}, \ldots,\left(\lambda^{1}\right)^{\prime}\right)$ and $\mathbf{s}^{\prime}=\left(-s_{\ell}, \ldots,-s_{1}\right)$.

\subsubsection{Abaci representation}

A charged $\ell$-partition $|\lambda, \mathbf{s}\rangle$ can also be represented by the $\mathbb{Z}$-graded $\ell$-abacus

$$
\mathcal{A}(\lambda, \mathbf{s})=\left\{\left(j, \lambda_{k}^{j}+s_{j}-k+1\right) ; j \in\{1, \ldots, \ell\}, k \in \mathbb{Z}_{\geq 1}\right\}
$$


where $\lambda_{k}^{j}$ denotes the $k$-th part of $\lambda^{j}$. In the rest of this paper, we will sometimes identify $|\lambda, \mathbf{s}\rangle$ with $\mathcal{A}(\lambda, \mathbf{s})$.

Example 2.2 With $|\lambda, \mathbf{s}\rangle$ as in Example 2.1, we get the following corresponding abacus

$$
\begin{aligned}
& \mathcal{A}(\boldsymbol{\lambda}, \mathbf{s}) \\
& \quad=\left\{\begin{array}{l}
\ldots,(2,-5),(2,-4),(2,-3),(2,-2),(2,-1),(2,0),(2,2),(2,3), \\
\ldots,(1,-5),(1,-4),(1,-3),(1,-1),(1,1)
\end{array}\right\}
\end{aligned}
$$

which we picture as follows, by putting a (black) bead at position $\left(j, \lambda_{k}^{j}+s_{j}-k+1\right)$ where $k \in \mathbb{Z}_{\geq 1}$ and $j \in\{1, \ldots, \ell\}$ is the row index (numbered from bottom to top):

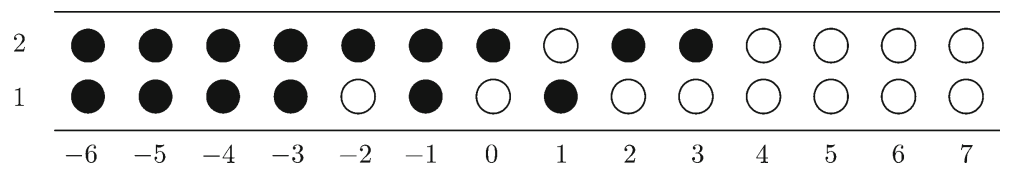

Note that the conjugate a multipartition can be easily described on the abacus: it suffices to rotate it by 180 degrees around the point of coordinates $\left(\frac{1}{2}, \frac{\ell}{2}\right)$ and swap the roles of the white and black beads.

Using the abaci realisation of charged multipartitions, we define below a bijection

$$
\begin{aligned}
\tau:\left\{|\lambda, s\rangle ; \lambda \in \Pi_{1}\right\} & \stackrel{\sim}{\longrightarrow}\left\{|\lambda, \mathbf{s}\rangle ; \lambda \in \Pi_{\ell},|\mathbf{s}|=s\right\} \\
\mathcal{A}(\lambda, s) & \longmapsto
\end{aligned}
$$

which can be seen as a twisted version of taking the $\ell$-quotient and the $\ell$-core of a partition, see [28, Chapter 1]. However, unlike the usual $\ell$-quotient and $\ell$-core, $\tau$ and $\tau^{-1}$ will depend not only on $\ell$, but also on $e$.

Writing down the Euclidean division first by $e \ell$ and then by $e$, one can decompose any $n \in \mathbb{Z}$ as $n=-z(n) e \ell+(y(n)-1) e+(x(n)-1)$ with $z(n) \in \mathbb{Z}, 1 \leq y(n) \leq \ell$ and $1 \leq x(n) \leq e$. We can then associate to each pair $(1, c) \in\{1\} \times \mathbb{Z}$ the pair $\tau(1, d)=(j, d) \in\{1, \ldots, \ell\} \times \mathbb{Z}$ where

$$
j=y(-c) \text { and } d=-(x(-c)-1)+e z(-c) .
$$

In particular, $\tau$ sends the bead in position $(1, c)$ into the rectangle $z(-c)$, on the row $y(-c)$ and column $x(-c)$ (numbered from right to left within each rectangle).

The map $\tau$ is bijective and we can see $\tau^{-1}$ as the following procedure:

(1) Divide the $\ell$-abacus into rectangles of size $e \times \ell$, where the $z$-th rectangle $(z \in \mathbb{Z})$ contains the positions $(j, d)$ for all $1 \leq j \leq \ell$ and $-e+1+z e \leq d \leq z e$.

(2) Relabel each $(j, d)$ by the second coordinate of $\tau^{-1}(j, d)$, see Fig. 1 for an example.

(3) Replace the newly indexed beads on a 1-abacus according to this new labeling.

Explicitly, $\tau$ and $\tau^{-1}$ verify the following formulas:

$$
\begin{aligned}
\tau(1, c) & =\left(-\left\lfloor\frac{-c}{e}\right\rfloor \bmod \ell+1,(-c \bmod e)+e\left\lfloor\frac{-c}{e \ell}\right\rfloor\right) \\
& =\left(\frac{-c-(-c \bmod e)}{e} \bmod \ell+1,(-c \bmod e)-e\left(\frac{-c-(-c \bmod e \ell)}{e \ell}\right)\right)
\end{aligned}
$$


Fig. 1 Relabelling bead positions in the $\ell$-abacus according to $\tau^{-1}$, for $\ell=4$ and $e=3$

\begin{tabular}{|c|c|c|c|c|c|c|c|}
\hline \multicolumn{4}{|c|}{$z=0$} & \multicolumn{4}{|c|}{$z=1$} \\
\hline$\ldots$ & -11 & -10 & -9 & 1 & 2 & 3 & $\cdots$ \\
\hline$\ldots$ & -8 & -7 & -6 & 4 & 5 & 6 & $\ldots$ \\
\hline$\ldots$ & -5 & -4 & -3 & 7 & 8 & 9 & $\ldots$ \\
\hline$\ldots$ & -2 & -1 & 0 & 10 & 11 & 12 & $\ldots$ \\
\hline-3 & -2 & -1 & 0 & 1 & 2 & 3 & 4 \\
\hline
\end{tabular}

and

$$
\begin{aligned}
\tau^{-1}(j, d) & =\left(1,-(-d \bmod e)-e(j-1)+e \ell\left\lfloor\frac{-d}{e}\right\rfloor\right) \\
& =(1, e(1-j)+\ell d-(\ell+1)(-d \bmod e)) .
\end{aligned}
$$

Example 2.3 We go back to Example 2.2. The actions of $\tau$ and $\tau^{-1}$ are represented below.
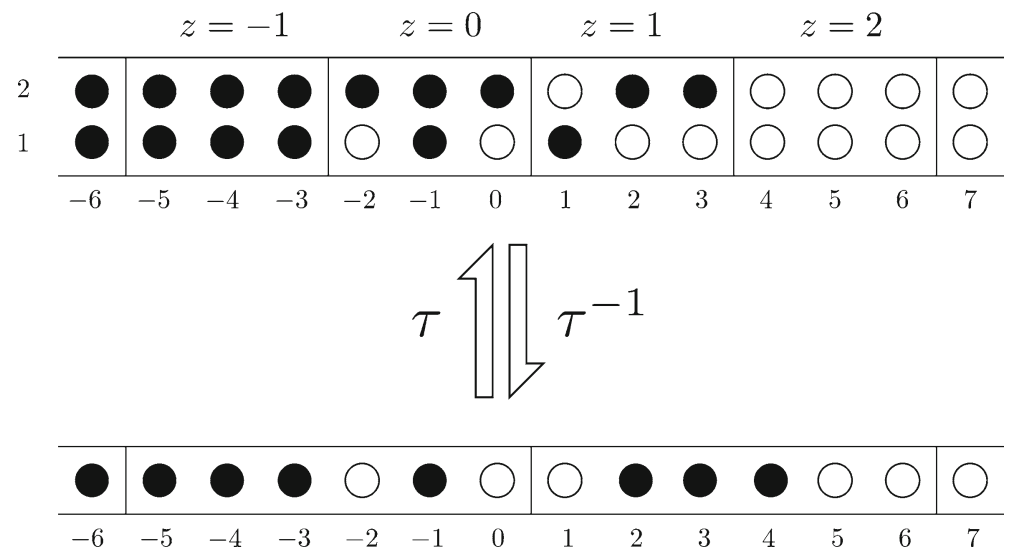

\subsubsection{Wedges}

Let $P(s)$ denote the set of sequences of integers $\boldsymbol{\alpha}=\left(\alpha_{1}, \alpha_{2}, \ldots\right)$ such that $\alpha_{k}=$ $s-k+1$ for $k$ sufficiently large, and set

$$
P_{>}(s)=\left\{\left(\alpha_{1}, \alpha_{2}, \ldots\right) \in P(s) \mid \alpha_{k}>\alpha_{k+1} \text { for all } k \in \mathbb{Z}_{\geq 1}\right\}
$$

Definition 2.4 An elementary wedge (respectively ordered wedge) is a formal element $u_{\alpha}=u_{\alpha_{1}} \wedge u_{\alpha_{2}} \wedge \ldots$ where $\boldsymbol{\alpha} \in P(s)$ (respectively $\boldsymbol{\alpha} \in P_{>}(s)$ ).

Let $|\lambda, \mathbf{s}\rangle$ be a charged $\ell$-partition with $|\mathbf{s}|=s$, and let $\lambda=\left(\lambda_{1}, \lambda_{2}, \ldots\right)$ be the partition such that $\tau(|\lambda, s\rangle)=|\lambda, \mathbf{s}\rangle$. Set $\boldsymbol{\beta}=\left(\beta_{1}, \beta_{2}, \ldots\right)$ where $\beta_{k}=\lambda_{k}+s-k+1$ 
for all $k \in \mathbb{Z}_{\geq 1}$. In other terms, $\boldsymbol{\beta}$ is the sequence of integers indexing the beads of $\mathcal{A}(\lambda, s)$.

We clearly have $\boldsymbol{\beta} \in P_{>}(s)$, so we can consider the elementary wedge $u_{\boldsymbol{\beta}}$. In fact, we will also identify $|\lambda, \mathbf{s}\rangle$ with $u_{\beta}$.

To sum up, we will allow the following identifications:

$$
|\lambda, \mathbf{s}\rangle \longleftrightarrow \mathcal{A}(\lambda, \mathbf{s}) \stackrel{\tau}{\longleftrightarrow} \mathcal{A}(\lambda, s) \longleftrightarrow u_{\beta} \longleftrightarrow|\lambda, s\rangle .
$$

We will denote

$$
B_{\mathbf{s}}=\left\{|\lambda, \mathbf{s}\rangle ; \lambda \in \Pi_{\ell}\right\} \text { for an } \ell \text {-charge } \mathbf{s}
$$

and

$$
B_{s}=\{|\lambda, s\rangle ; \lambda \in \Pi\}=\bigsqcup_{|\mathbf{s}|=s} B_{\mathbf{s}} .
$$

\subsection{Fock space as $\mathcal{U}_{q}^{\prime}\left(\widehat{\mathfrak{s l}}_{e}\right)$-module}

Let $q$ be an indeterminate.

\subsubsection{The JMMO Fock space}

Fix an $\ell$-charge $\mathbf{s}$. The Fock space associated to $\mathbf{s}$ is the $\mathbb{Q}(q)$-vector space $\mathcal{F}_{\mathbf{s}}$ with basis $B_{\mathbf{s}}$.

Theorem 2.5 (Jimbo et al. [14]). The space $\mathcal{F}_{\mathbf{s}}$ is an integrable $\mathcal{U}_{q}^{\prime}\left(\widehat{\mathfrak{s l}_{e}}\right)$-module of level $\ell$.

The action of the generators of $\mathcal{U}_{q}^{\prime}\left(\widehat{\mathfrak{s l}_{e}}\right)$ depends on $\mathbf{s}$ and $e$, and is given explicitly in terms of addable/removable boxes, see e.g. [7, Section 6.2]. In turn, this induces a $\mathcal{U}_{q}^{\prime}\left(\widehat{\mathfrak{s l}}_{e}\right)$-crystal structure (also called Kashiwara crystal) [15], usually encoded in a graph $\mathscr{B}_{\mathbf{s}}$, whose vertices are the elements of $B_{\mathbf{s}}$, and with colored arrows representing the action of the Kashiwara operators. An explicit (recursive) formula for computing this graph was first given in [14]: two vertices are joined by an arrow if and only if one is obtained from the other by adding/removing a good box, see [7, Section 6.2] for details.

It has several connected components, each of which parametrised by its only source vertex, or highest weight vertex. The decomposition of this graph in connected components reflects the decomposition of $\mathcal{F}_{\mathrm{s}}$ into irreducible representations (which exists because $\mathcal{F}_{\mathrm{s}}$ is integrable according to Theorem 2.5).

\subsubsection{Uglov's wedge space}

Denote $\mathcal{F}_{s}$ the $\mathbb{Q}(q)$-vector space spanned by the elementary wedges, and subject to the straightening relations defined in [27, Proposition 3.16]. This is called the space 
of semi-infinite q-wedges, or simply the wedge space, and the elements of $\mathcal{F}_{s}$ will be called wedges.

Using the straightening relations, a $q$-wedge can be expressed as a $\mathbb{Q}(q)$-linear combination of ordered wedges. In fact, one can show (see [27, Proposition 4.1]) that the set of ordered wedges $B_{s}$ forms a basis of $\mathcal{F}_{s}$.

Remark 2.6 (1) The terminology "wedge space" is justified by the original construction of this vector space in the level one case by Kashiwara, Miwa and Stern [16]. In this context, one can first construct a quantum version of the usual $k$-fold wedge product (or exterior power) $\Lambda^{k} V$, where $V$ is the natural $\mathcal{U}_{q}^{\prime}\left(\widehat{\mathfrak{s l}}_{e}\right)$-representation (an affinisation of $\mathbb{C}^{\ell} \otimes \mathbb{C}^{\ell}$ ). The space $\mathcal{F}_{S}$ is then defined as the projective limit (taking $k \rightarrow \infty$ ) of $\Lambda^{k} V$. In the higher level case, the analogous construction was achieved by Uglov [27].

(2) Using the bijection between ordered wedges and partitions charged by $s$, one can see $\mathcal{F}_{S}$ as a level 1 Fock space, whence the notation. In fact, it is sometimes called the fermionic Fock space.

Theorem 2.7 The wedge space $\mathcal{F}_{s}$ is an integrable $\mathcal{U}_{q}^{\prime}\left(\widehat{\mathfrak{s l}_{e}}\right)$-module of level $\ell$.

Proof The identification $B_{s}=\left\{u_{\boldsymbol{\beta}} \in \mathcal{F}_{s} \mid \beta \in P_{>}(s)\right\}=\left\{|\lambda, \mathbf{s}\rangle \in \mathcal{F}_{\mathbf{s}} ;|\mathbf{s}|=s\right\}=$ $\bigsqcup_{|\mathbf{s}|=s} B_{\mathbf{S}}$ of Sect. 2.1 .3 yields the $\mathbb{Q}(q)$-vector space decomposition

$$
\mathcal{F}_{s}=\bigoplus_{|\mathbf{s}|=s} \mathcal{F}_{\mathbf{s}}
$$

By Theorem 2.2.1, this decomposition still holds as integrable $\mathcal{U}_{q}^{\prime}\left(\widehat{\mathfrak{s l}_{e}}\right)$-module.

\section{The Heisenberg action}

It turns out that the wedge space has some additional structure, namely that of a $\mathcal{H}$ module, where $\mathcal{H}$ is the (quantum) Heisenberg algebra. This has been first observed by Uglov [27], generalising some results of Lascoux, Leclerc and Thibon [17]. Let us recall the definition of this algebra.

\subsection{The action of the bosons}

Let us start by recalling the definition of the quantum Heisenberg algebra.

Definition 3.1 The (quantum) Heisenberg algebra is the unital $\mathbb{Q}(q)$-algebra $\mathcal{H}$ with generators $p_{m}, m \in \mathbb{Z}^{\times}$and defining relations

$$
\left[p_{m}, p_{m^{\prime}}\right]=\delta_{m,-m^{\prime}} m \frac{1-q^{-2 m e}}{1-q^{-2 m}} \times \frac{1-q^{2 m \ell}}{1-q^{2 m}}
$$

for $m, m^{\prime} \in \mathbb{Z}^{\times}$. The elements $p_{m}$ are called bosons. 
Note that this is a $q$-deformation of the usual Heisenberg algebra with relations $\left[p_{m}, p_{m^{\prime}}\right]=\delta_{m,-m^{\prime}} m$.

Theorem 3.2 The formula

$$
\begin{aligned}
& p_{m}\left(u_{\boldsymbol{\beta}}\right)=\sum_{k \geq 1} u_{\beta_{1}} \wedge \ldots \wedge u_{\beta_{k-1}} \wedge u_{\beta_{k-e \ell m}} \wedge u_{\beta_{k+1}} \wedge \ldots \\
& \qquad \text { for } u_{\beta} \in \mathcal{F}_{S} \text { and } m \in \mathbb{Z}^{\times}
\end{aligned}
$$

endows $\mathcal{F}_{s}$ with the structure of an $\mathcal{H}$-module.

For a proof, we refer to [27, Proposition 4.4 and 4.5]. This is quite technical and is done in two distinct steps, the second of which requires the notion of asymptotic wedge. This will be crucial in Sect. 4.

Corollary 3.3 The action of the bosons preserves the level $\ell$ Fock spaces $\mathcal{F}_{\mathbf{s}}$ for $|\mathbf{s}|=s$.

One can use the explicit action of $p_{m}$ on $\mathcal{F}_{s}$ to show that it preserves the $\ell$-charges s, or rely on Uglov's argument [27, Section 4.3]. As a consequence of this corollary, $\mathcal{F}_{s}=\bigoplus_{|\mathbf{s}|=s} \mathcal{F}_{\mathbf{s}}$ also holds as $\mathcal{H}$-module decomposition.

\subsection{Some notations and definitions}

For two partitions $\lambda$ and $\mu$, denote $\lambda+\mu$ the reordering of $\left(\lambda_{1}, \mu_{1}, \lambda_{2}, \mu_{2}, \ldots\right)$ and $k \lambda$ the partition $\left(\lambda_{1}^{k} \cdot \lambda_{2}^{k} \ldots\right)$. Extend these notations to $\ell$-partitions by doing these operations coordinatewise.

Note that this is simply the conjugation of the usual notation for adding partitions or multiplying by an integer.

Example 3.4 We have

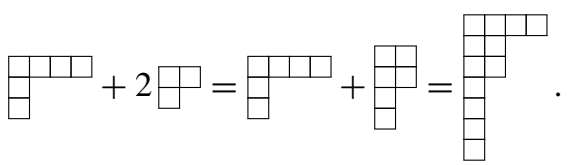

We also define the notion of asymptotic charges, which will mostly be useful in Sect. 4.

Definition 3.5 Let $j_{0} \in\{1, \ldots, \ell\}$. A wedge $u_{\boldsymbol{\beta}} \in \mathcal{F}_{S}$ is called $j_{0}$-asymptotic if the corresponding charged multipartition $|\lambda, \mathbf{s}\rangle$ verifies $s_{j_{0}}-s_{j} \geq|\lambda|$ for all $j \neq j_{0}$.

\subsection{The Heisenberg crystal}

A notion of crystal for the Heisenberg algebra, or $\mathcal{H}$-crystal, has been indepedently introduced by Losev [19] and the author [9]. Explicit formulas for computing this 
crystal have been given in some particular cases: asymptotic in [19] and doubly highest weight in [9].

Recall the definition of the $\mathcal{H}$-crystal according to [9]. This requires the crystal level-rank duality exposed in "Appendix".

Definition 3.6 Let $|\lambda, \mathbf{s}\rangle \in B(s)$, which we identify with its level-rank dual charged $e$-partition. We call $|\lambda, \mathbf{s}\rangle$ a doubly highest weight vertex if it is a highest weight vertex simultaneously in the $\mathcal{U}_{q}^{\prime}\left(\widehat{\mathfrak{s l}_{e}}\right)$-crystal and in the $\mathcal{U}_{p}^{\prime}\left(\widehat{\mathfrak{s l}_{\ell}}\right)$-crystal.

Some important properties of doubly highest weight vertices are exposed in [9, Section 5.2]. In particular, an element $|\lambda, \mathbf{s}\rangle$ is a doubly highest weight vertex if and only if it has a totally $e$-periodic $\ell$-abacus and a totally $\ell$-periodic $e$-abacus, according to a result by Jacon and Lecouvey [12], see the definition therein. Moreover, every bead of a given period encodes a same part size in $\lambda$, and one can define the partition $\kappa=\kappa(|\lambda, \mathbf{s}\rangle)$ as $\left(\kappa_{1}, \kappa_{2} \ldots\right)$ where $\kappa_{k}$ is the part encoded by the $k$-th period.

Definition 3.7 Let $\sigma \in \Pi$. The Heisenberg crystal operator $\tilde{b}_{\sigma}$ (respectively $\tilde{b}_{-\sigma}$ ) is the uniquely determined map $B_{S} \rightarrow B_{S},|\lambda, \mathbf{s}\rangle \mapsto|\boldsymbol{\mu}, \mathbf{s}\rangle$ (respectively $B_{S} \rightarrow B_{S} \sqcup\{0\}$ ) such that

(1) if $|\lambda, \mathbf{s}\rangle$ is a doubly highest weight vertex, then $|\boldsymbol{\mu}, \mathbf{s}\rangle$ is obtained from $|\lambda, \mathbf{s}\rangle$ by shifting the $k$-th period of $\ell$-abaci of $|\lambda, \mathbf{s}\rangle$ by $\sigma_{k}$ steps to the right (respectively to the left when possible, and $\tilde{b}_{-\sigma}|\lambda, \mathbf{s}\rangle=0$ otherwise),

(2) it commutes with the Kashiwara crystal operators of $\mathcal{U}_{q}^{\prime}\left(\widehat{\mathfrak{s l}_{e}}\right)$ and of $\mathcal{U}_{p}^{\prime}\left(\widehat{\mathfrak{s l}_{\ell}}\right)$.

Definition 3.8 We say that $|\lambda, \mathbf{s}\rangle \in B_{s}$ is a highest weight vertex for $\mathcal{H}$ if $\tilde{b}_{-\sigma}|\lambda, \mathbf{s}\rangle=0$ for all $\sigma \in \Pi$.

Note that each $\tilde{b}_{ \pm \sigma}$ is well defined since (2) allows to define $\tilde{b}_{ \pm \sigma}|\lambda, \mathbf{s}\rangle$ even when $|\lambda, \mathbf{s}\rangle$ is not a doubly highest weight vertex. Moreover, [9, Theorem 7.6] claims that in the asymptotic case, the Heisenberg crystal operators coincide with the combinatorial maps introduced by Losev [19]. Note also that the definition of $\kappa$ for doubly highest weight vertices induces (by (2) of Definition 3.7) a surjective map $\kappa: B_{s} \rightarrow \Pi$ such that $\tilde{b}_{-\kappa(|\lambda, \mathbf{s}\rangle)}|\lambda, \mathbf{s}\rangle$ is a highest weight vertex for $\mathcal{H}$ for all $\lambda \in \Pi_{\ell}$.

In order to have a description of the $\mathcal{H}$-crystal similar to the $\mathcal{U}_{q}^{\prime}\left(\widehat{\mathfrak{s t}_{e}}\right)$-crystal, see Fig. 2, we wish to define the $\mathcal{H}$-crystal as a graph whose arrows have minimal length. Therefore, we define the following maps

$$
\tilde{b}_{1, c}=\tilde{b}_{\eta} \tilde{b}_{-\kappa} \text { and } \tilde{b}_{-1, d}=\tilde{b}_{\theta} \tilde{b}_{-\kappa}
$$

where $\eta=\kappa \sqcup\{\gamma\}$ with $\gamma=(a, b)$ being the addable box of $\kappa$ verifying $b-a=c$ and where $\theta=\kappa \backslash\{\gamma\}$ with $\gamma=(a, b)$ being the removable box of $\kappa$ verifying $b-a=d$,

Definition 3.9 The $\mathcal{H}$-crystal of the wedge space $\mathcal{F}_{S}$ is the graph $\mathscr{C}_{s}$ with

(1) vertices: all charged $\ell$-partitions $\mid \lambda$, s $\rangle$ with $\lambda \in \Pi_{\ell}$ and $|\mathbf{s}|=s$.

(2) arrows : $|\lambda, \mathbf{s}\rangle \stackrel{c}{\longrightarrow}|\boldsymbol{\mu}, \mathbf{s}\rangle$ if $|\boldsymbol{\mu}, \mathbf{s}\rangle=\tilde{b}_{1, c}|\lambda, \mathbf{s}\rangle$. 


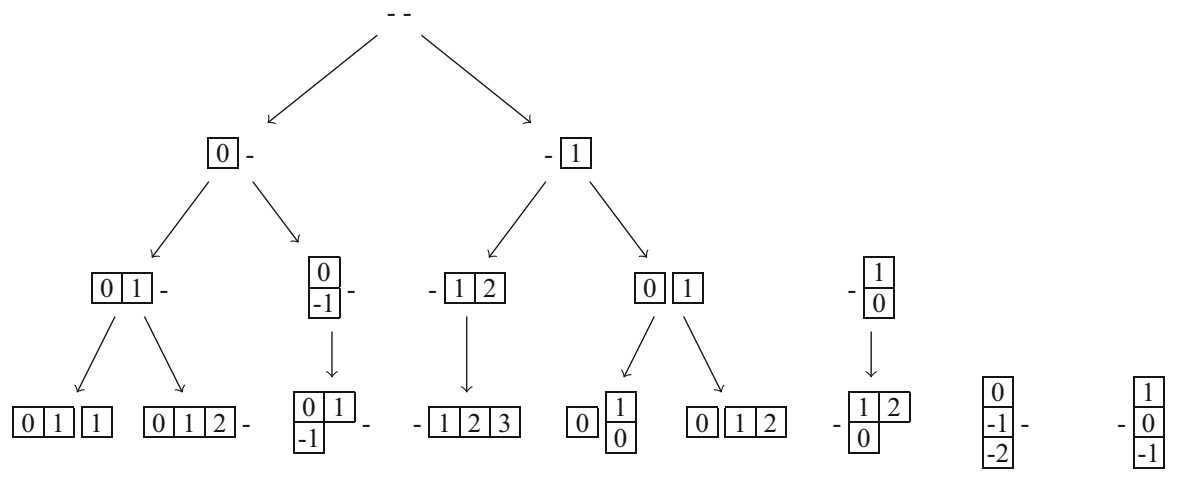

Fig. 2 The beginning of the Kashiwara crystal graph $\mathscr{B}_{\mathbf{s}}$ of $\mathcal{F}_{\mathbf{s}}$ for $\ell=2, e=3$ and $\mathbf{s}=(0,1)$

Remark 3.10 - Note that $\tilde{b}_{\eta} \tilde{b}_{-\kappa}=\tilde{b}_{\varepsilon} \tilde{b}_{-\omega}$ for all $\omega \subseteq \kappa$ and $\varepsilon \subseteq \eta$ such that $\varepsilon / \omega=\eta / \kappa$, so that the map $\tilde{b}_{1, c}$ depends only on $\eta / \kappa$.

- By definition, each $\tilde{b}_{ \pm 1, c}$ is a composition of maps $\tilde{b}_{ \pm \sigma}$. Conversely, each $\tilde{b}_{\sigma}$ is a composition of maps $\tilde{b}_{1, c}$, see [9, Formula (6.17)]. We will also call the maps $\tilde{b}_{1, c}$ Heisenberg crystal operators.

Proposition 3.11 (1) The $\mathcal{H}$-module decomposition $\mathcal{F}_{s}=\bigoplus_{|\mathbf{s}|=s} \mathcal{F}_{\mathbf{s}}$ induces a decomposition of $\mathscr{C}_{s}$.

(2) Each connected component of $\mathscr{C}_{s}$ is isomorphic to the Young graph.

(3) A vertex $|\lambda, \mathbf{s}\rangle$ is source in $\mathscr{C}_{s}$ if and only if $|\lambda, \mathbf{s}\rangle$ is a highest weight vertex for $\mathcal{H}$.

(4) The depth of an element $\mid \lambda$, $\mathbf{s}\rangle$ in $\mathscr{C}_{s}$ is $\mid \kappa(|\lambda, \mathbf{s}\rangle) \mid$.

Proof We know from Definition 3.7 that the action of the crystal Heisenberg operators preserves the multicharge, proving (1). In particular, there is a notion of $\mathcal{H}$-crystal for $\mathcal{F}_{\mathbf{s}}$, which we denote $\mathscr{C}_{\mathbf{s}}$. Moreover, the $\mathcal{H}$-crystal is characterised by being the preimage of a $\mathcal{U}_{q}\left(\mathfrak{s l}_{\infty}\right)$-crystal on the set of partitions under a certain bijection, depending on $\kappa$, see [9, Remark 6.16]. The $\mathcal{U}_{q}\left(\mathfrak{s l}_{\infty}\right)$-crystal is exactly the Young graph on partitions, see Fig. 3, where the arrows are colored by the contents of the added boxes, which proves (2). In fact, the bijection from the Young graph to a given connected component, parametrised by its source vertex $|\bar{\lambda}, \mathbf{s}\rangle$ is given by $\sigma \mapsto \tilde{b}_{\sigma}|\bar{\lambda}, \mathbf{s}\rangle$, and its inverse is $|\lambda, \mathbf{s}\rangle \mapsto \kappa(|\lambda, \mathbf{s}\rangle)$. Point (3) is clear by definition, and (4) follows from the definition of $\kappa$.

\section{Canonical bases and Schur functions}

\subsection{The Boson-Fermion correspondence}

Denote $\Lambda$ the algebra of symmetric functions, that is, the projective limit of the $\mathbb{Q}(q)$ algebras of symmetric polynomials in finitely many indeterminates [21, Chapter 1]:

$$
\Lambda=\mathbb{Q}(q)\left[X_{1}, X_{2}, \ldots\right]^{\mathfrak{S}_{\infty}}
$$


Fig. 3 The beginning of the Young graph

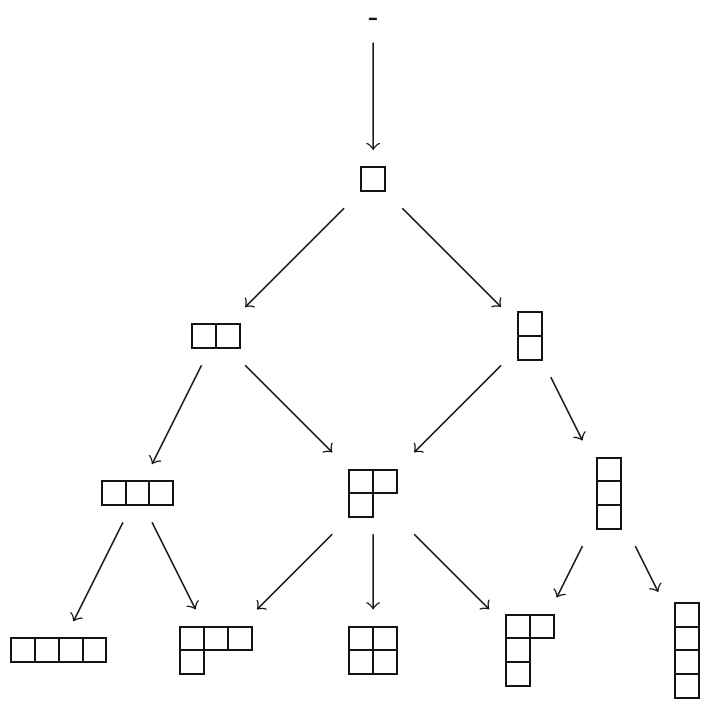

The space $\Lambda$ has several natural linear bases, among which:

- the monomial functions $\left\{M_{\sigma} ; \sigma \in \Pi\right\}$ where $M_{\sigma}=\sum_{\pi} X_{1}^{\pi_{1}} X_{2}^{\pi_{2}} \ldots$ where the sum runs over all permutations $\pi$ of $\sigma$,

- the complete functions $\left\{H_{\sigma} ; \sigma \in \Pi\right\}$ where $H_{\sigma}=H_{\sigma_{1}} H_{\sigma_{2}} \ldots$ and $H_{m}=$ $\sum_{k_{1} \leq \cdots \leq k_{m}} X_{k_{1}} \ldots X_{k_{m}}$ for $r \in \mathbb{N}$,

- the power sums $\left\{P_{\sigma} ; \sigma \in \Pi\right\}$ where $P_{\sigma}=P_{\sigma_{1}} P_{\sigma_{2}} \ldots$ and $P_{m}=\sum_{k \geq 1} X_{k}^{r}$ for $r \in \mathbb{N}$,

- the Schur functions $\left\{S_{\sigma} ; \sigma \in \Pi\right\}$ where $S_{\sigma}=\sum_{\pi \in \Pi} K_{\sigma, \pi} M_{\pi}$ where $K_{\sigma, \pi}$ are the Kostka numbers, see [25, Chapter 7].

The expansion of $H_{m}$ in the basis of the power sums is given by

$$
H_{m}=\sum_{\substack{\pi \in \Pi \\|\pi|=m}} \frac{1}{z_{\pi}} P_{\pi},
$$

where $z_{\pi}=\Pi_{k>0} k^{\alpha_{k}} \alpha_{k}$ with $\alpha_{k}=\pi_{k}^{\prime}-\pi_{k+1}^{\prime}$, for all $\pi \in \Pi$. Moreover, by duality, the Kostka numbers also appear as the coefficients of the complete functions in the basis of the Schur functions:

$$
H_{\sigma}=\sum_{\pi \in \Pi} K_{\pi, \sigma} S_{\pi} .
$$

By a result of Miwa, Jimbo and Date [22], there is a vector space isomorphism

$$
\begin{aligned}
\mathcal{F}_{s} & \stackrel{\sim}{\longrightarrow} \Lambda \\
|\lambda, s\rangle & \longmapsto S_{\lambda},
\end{aligned}
$$


called the Boson-Fermion correspondence. In fact, when refering to the symmetric realisation $\Lambda$, one sometimes uses the term bosonic Fock space, as opposed to the fermionic, antisymmetric definition of $\mathcal{F}_{s}$. The following result is [24, Section 4.2 and Proposition 4.6].

Proposition 4.1 There is a $\mathcal{H}$-module isomorphism $\mathcal{F}_{s} \simeq \Lambda$, where, at $q=1$, the action of $p_{m}$ on $\mathcal{F}_{s}$ is mapped to the multiplication by $P_{m}$ on $\Lambda$.

In general, the action of $p_{m}$ is mapped to a $q$-deformation of the multiplication by $P_{m}$, and $p_{-m}$ to a $q$-deformation of the derivation with respect to $P_{m}$, see [17, Section 5] and [27, Section 5.1].

\subsection{Action of the Schur functions}

In [18], Leclerc and Thibon studied the action of the Heisenberg algebra on level 1 Fock spaces in order to give an analogue of Lusztig's version [20] of the Steinberg tensor product theorem. Their idea was to use a different basis of $\Lambda$ to compute the Heisenberg action in a simpler way, namely that of Schur functions. This result has been generalised to the level $\ell$ case by Iijima in a particular case [11].

Independently, the Schur functions have been used by Shan and Vasserot to categorify the Heisenberg action in the context of Cherednik algebras. More precisely, they constructed a functor on the category $\mathcal{O}$ for cyclotomic rational Cherednik algebras corresponding to the multiplication by a Schur function on the bosonic Fock space $\Lambda$ [24, Proposition 5.13].

The aim of this section is to use Iijima's result to recover in a direct, simple way the results of $[9,19]$ and, by doing so, bypassing Shan and Vasserot's categorical constructions.

\subsubsection{Canonical bases of the Fock space}

In the early nineties, Kashiwara and Lusztig have independently introduced the notion of canonical bases for irreducible highest weight representations of quantum groups, see e.g. [15]. They are characterised by their invariance under a certain involution. Uglov [27] has proved an analogous result for the Fock spaces $\mathcal{F}_{\mathbf{s}}$, even though it is no longer irreducible. We recall the definition of the involution on $\mathcal{F}_{s}$ and Uglov's theorem.

For any $r \in \mathbb{N}$ and $t_{1}, \ldots, t_{r} \in \mathbb{Z}^{r}$, let $\iota\left(t_{1}, \ldots, t_{r}\right)=\sharp\left\{\left(k, k^{\prime}\right) \mid k<k^{\prime}\right.$ and $\left.t_{k}=t_{k^{\prime}}\right\}$ that is, the number of repetitions in $\left(t_{1}, \ldots, t_{r}\right)$.

Definition 4.2 The bar involution is the $\mathbb{Q}(q)$-vector space automorphism

$$
\begin{aligned}
& \mathcal{F}_{s} \longrightarrow \mathcal{F}_{s} \\
& q \longmapsto \bar{q}=q^{-1} \\
& u_{\beta} \longmapsto \overline{u_{\beta}}
\end{aligned}
$$


with

$$
\begin{aligned}
\overline{u_{\beta}} & =\overline{u_{\beta_{1}} \wedge \ldots \wedge u_{\beta_{r}}} \wedge u_{\beta_{r+1}} \wedge \ldots \\
& =(-q)^{\iota\left(y_{1}, \ldots, y_{r}\right)} q^{\iota\left(x_{1}, \ldots, x_{r}\right)}\left(u_{\beta_{r}} \wedge \ldots \wedge u_{\beta_{1}}\right) \wedge u_{\beta_{r+1}} \wedge \ldots
\end{aligned}
$$

where $x_{k}=x\left(\beta_{k}\right)$ and $y_{k}=y\left(\beta_{k}\right)$ for all $k=1, \ldots, r$ according to the notation of Sect. 2.1.2.

The bar involution behaves nicely on the wedge space, in particular it preserves the level $\ell$ Fock spaces $\mathcal{F}_{\mathbf{s}}$ for $|\mathbf{s}|=s$, and commutes with the bosons $p_{m}$, this is $[27$, Section 4.4]. We can now state Uglov's result, that is derived from the fact that the matrix of the bar involution is unitriangular.

Theorem 4.3 Let $\mathbf{s} \in \mathbb{Z}^{\ell}$ such that $|\mathbf{s}|=s$. There exist unique bases $\mathcal{G}^{+}=$ $\left\{G^{+}(\lambda, \mathbf{s}) ; \lambda \in \Pi_{\ell}\right\}$ and $\mathcal{G}^{-}=\left\{G^{-}(\lambda, \mathbf{s}) ; \lambda \in \Pi_{\ell}\right\}$ of $\mathcal{F}_{\mathbf{s}}$ such that, for $b \in\{+,-\}$,

(1) $\overline{G^{b}(\lambda, \mathbf{s})}=G^{\mathrm{b}}(\lambda, \mathbf{s})$

(2) $G^{b}(\lambda, \mathbf{s})=|\lambda, \mathbf{s}\rangle \bmod q^{b 1} \mathcal{L}^{b}$ where $\mathcal{L}^{b}=\bigoplus_{\lambda \in \Pi_{\ell}} \mathbb{Q}\left[q^{b 1}\right]|\lambda, \mathbf{s}\rangle$.

This result is compatible with Kashiwara's crystal theory. More precisely, each integrable irreducible highest weight $\mathcal{U}_{q}^{\prime}\left(\widehat{\mathfrak{s l}}_{e}\right)$-representation is contained in $\mathcal{F}_{s}$ for some $s$, by taking the span of the vectors $|\emptyset, \mathbf{s}\rangle$ for $|\mathbf{s}|=s$, and it is proved in [27, Section 4.4] that the bases $\mathcal{G}^{b}$ restricted to $\mathcal{U}_{q}^{\prime}\left(\widehat{\mathfrak{s l}_{e}}\right)|\emptyset, \mathbf{s}\rangle$ coincide with Kashiwara's lower and upper canonical bases. Therefore, we also call $\mathcal{G}^{b}$ the (lower or upper if $b=-$ or + respectively) canonical basis of $\mathcal{F}_{\mathbf{s}}$.

\subsubsection{Schur functions in the asymptotic case}

Define the following operators on $\mathcal{F}_{s}$

$$
h_{m}=\sum_{|\pi|=m} \frac{1}{z_{\pi}} p_{\pi}, \quad h_{\sigma}=h_{\sigma_{1}} h_{\sigma_{2}} \ldots \quad \text { and } \quad s_{\sigma}=\sum_{\pi \in \Pi} K_{\pi, \sigma}^{-1} h_{\pi} .
$$

where $K_{\pi, \sigma}^{-1}$ are the inverse Kostka numbers, that is, the entries of the inverse of the matrix of Kostka numbers. By Proposition 4.1, at $q=1$, the action of $h_{\sigma}$ (respectively $s_{\sigma}$ ) corresponds to the multiplication by a complete function (respectively Schur function) on $\Lambda$ through the Boson-Fermion correspondence.

Theorem 4.4 (1) The operators $s_{\sigma}$ induce maps $\tilde{s}_{\sigma}$ on $B_{s}$, preserving $B_{\mathbf{s}}$ for $|\mathbf{s}|=s$. (2) Let $|\lambda, \mathbf{s}\rangle \in \mathcal{F}_{\mathbf{s}}$ be j0-asymptotic. We have $\tilde{s}_{\sigma}|\lambda, \mathbf{s}\rangle=|\boldsymbol{\mu}, \mathbf{s}\rangle$ with $\boldsymbol{\mu}=\boldsymbol{\lambda}+\boldsymbol{e} \boldsymbol{\sigma}$ where $\sigma^{j}=\emptyset$ if $j \neq j_{0}$ and $\sigma^{j_{0}}=\sigma$, provided $|\boldsymbol{\mu}, \mathbf{s}\rangle$ is still $j_{0}$-asymptotic. In particular, $\tilde{s}_{\sigma}$ coincides with the Heisenberg crystal operator $\tilde{b}_{\sigma}$ in this case. 
Remark 4.5 When $\lambda=\emptyset$, this says that $\tilde{s}_{\sigma}$ shifts the $e$ rightmost beads in the $j_{0}$-th runner of $\mathcal{A}(\lambda, \mathbf{s})$, and one recovers Losev's result, see for instance [9, Example 7.3].

Proof In a general manner, we can identify a crystal map $B_{S} \rightarrow B_{S}$ with an operator on the wedge space $\mathcal{F}_{s}$ mapping an element of $\mathcal{G}^{b}$ to an element of $\mathcal{G}^{b}$, for $b \in\{+,-\}$. Indeed, an element $|\lambda, \mathbf{s}\rangle \in B_{s}$ can be identified with $G^{b}(\lambda, \mathbf{s})$. Another way to see this is to look at the action on $G^{b}(\lambda, \mathbf{s})$ and put $q=0$ or $q=\infty$ respectively in the resulting vector. This provides the identification because of Condition (2) of Theorem 4.3. As a matter of fact, the action of $s_{\sigma}$ on a canonical basis element turns out to have the desired form. Indeed, the version of [11, Theorem 4.12] in the $j_{0}$-asymptotic case ${ }^{1}$ states that

$$
s_{\sigma} G^{+}(\lambda, \mathbf{s})=G^{+}(\boldsymbol{\mu}, \mathbf{s})
$$

where $\boldsymbol{\mu}=\lambda+e \boldsymbol{\sigma}$ (with $\boldsymbol{\sigma}$ as in the statement of Theorem 4.4) provided:

$-\lambda^{j_{0}}$ is $e$-regular

$-|\lambda, \mathbf{s}\rangle$ and $|\boldsymbol{\mu}, \mathbf{s}\rangle$ are $j_{0}$-asymptotic.

Here, Iijima's original statement has been twisted by conjugation, because the levelrank duality used in his result is the reverse of that of the present paper. Thus, the notion of $e$-restricted multipartition is replaced by $e$-regular. Accordingly, we use the upper canonical basis instead of the lower one. This statement can be extended to an arbitrary $j_{0}$-asymptotic element $G^{+}(\boldsymbol{\lambda}, \mathbf{s}) \in \mathcal{F}_{S}$, that is, without the restriction that $\lambda^{j_{0}}$ is $e$-regular. To do this, for all $\lambda \in \Pi_{\ell}$, let $\lambda=\tilde{\lambda}+e \pi$ where $\tilde{\lambda}^{j_{0}}$ is $e$-regular and $\pi^{j}=\emptyset$ if $j \neq j_{0}$ and $\pi^{j_{0}}=\pi$. Then, for all $\sigma \in \Pi$,

$$
\begin{aligned}
s_{\sigma} G^{+}(\lambda, \mathbf{s}) & =s_{\sigma}\left(s_{\pi} G^{+}(\tilde{\lambda}, \mathbf{s})\right) \text { by the above formula } \\
& =s_{\tau} G^{+}(\tilde{\lambda}, \mathbf{s})
\end{aligned}
$$

where $\tau=\left(\sigma^{\prime}+\pi^{\prime}\right)^{\prime}$, which we can compute by the preceding formula. Note that $\tau$ is the common addition of $\sigma$ and $\pi$. For a general $j_{0}$-asymptotic vector $|\lambda, \mathbf{s}\rangle$, we write again $G^{+}(\boldsymbol{\mu}, \mathbf{s})=s_{\sigma} G^{+}(\lambda, \mathbf{s})$. As explained in the beginning of the proof, this induces a crystal map $\tilde{s}_{\sigma}: B_{s} \rightarrow B_{S}$, by additionnaly requiring that $\tilde{s}_{\sigma}$ commutes with the Kashiwara crystal operators. In fact, when $|\lambda, \mathbf{s}\rangle$ is $j_{0}$-asymptotic, the formula for $\tilde{s}_{\sigma}$ is precisely the formula for the Heisenberg crystal operator given in [19] (provided again that one twists by conjugating), which coincides with $\tilde{b}_{\sigma}$ by [9, Theorem 7.6]. This completes the proof.

As explained in the proof, with this approach, $\tilde{s}_{\sigma}|\lambda, \mathbf{s}\rangle$ is identified with $s_{\sigma} G^{+}(\lambda, \mathbf{s})$. The map $s_{\sigma}$ being an actual operator (on the vector space $\mathcal{F}_{S}$ ), this justifies the terminology "operator" for the maps $\tilde{b}_{\sigma}: B_{S} \rightarrow B_{S}$, thereby completing the analogy with the Kashiwara crystal operators.

\footnotetext{
${ }^{1}$ Note that Iijima's definition of asymptoticity differs slightly from that of the present paper: it is defined globally instead of locally.
} 


\section{Explicit description of the Heisenberg crystal}

In this section, we give the combinatorial formula for computing the Heisenberg crystal in full generality. This completes the results of [19], where the asymptotic case is treated, and of [9] where the case of doubly highest weight vertices (in the level-rank duality) is treated, see "Appendix" for details.

\subsection{Level $\ell$ vertical strips}

In the spirit of [14] and [6], we will express the action of the Heisenberg crystal operators in terms of adding/removing certain vertical strips.

For a given charged multipartition $|\lambda, \mathbf{s}\rangle$, we denote

$$
\begin{aligned}
& \mathscr{W}_{1}(\lambda, \mathbf{s})=\left\{(a, b, j) \in \mathbb{Z}_{>0} \times \mathbb{Z}_{\geq 1} \times\{1, \ldots, \ell\} \mid(a, b, j) \notin \lambda\right. \\
& \quad \text { and }(a, b-1, j) \in \lambda\} \\
& \mathscr{W}_{2}(\lambda, \mathbf{s})=\left\{(a, 1, j) \in \mathbb{Z}_{>0} \times\{1\} \times\{1, \ldots, \ell\} \mid(a, 1, j) \notin \lambda\right\}
\end{aligned}
$$

and $\mathscr{W}(\lambda, \mathbf{s})=\mathscr{W}_{1}(\lambda, \mathbf{s}) \sqcup \mathscr{W}_{2}(\lambda, \mathbf{s})$, so that $\mathscr{W}(\lambda, \mathbf{s})$ is the set of boxes directly to the right of $\lambda$ (considering that it has infinitely many parts of size zero).

Definition 5.1 Let $|\lambda, \mathbf{s}\rangle$ be a charged $\ell$-partition.

(1) A (level $\ell)$ vertical $e$-strip is a sequence of $e$ boxes $\gamma_{1}=\left(a_{1}, b_{1}, j_{1}\right), \ldots, \gamma_{e}=$ $\left(a_{e}, b_{e}, j_{e}\right)$ such that no horizontal domino appears, i.e. there is no $1 \leq i \leq e$ such that $a_{i+1}=a_{i}$ and $j_{i+1}=j_{i}$. Moreover, a vertical $e$-strip is called admissible if:

(a) The contents of $\gamma_{1}, \ldots, \gamma_{e}$ are consecutive, say $\mathfrak{c}\left(\gamma_{i}\right)=\mathfrak{c}\left(\gamma_{i+1}\right)+1$ for all $1 \leq i \leq e$.

(b) For all $1 \leq i<i^{\prime} \leq e$, we have $j_{i} \geq j_{i^{\prime}}$.

(2) The admissible vertical $e$-strips contained in $\mathscr{W}(\lambda, \mathbf{s})$ are denoted $\mathscr{V}(\lambda, \mathbf{s})$. For convenience, we might drop the word "admissible" in the following.

(3) Let $X \in \mathscr{V}(\boldsymbol{\lambda}, \mathbf{s}) . X$ is called addable if $X \cap \lambda=\emptyset$ and $\lambda \sqcup X$ is still an $\ell$-partition.

Remark 5.2 This is a generalisation to multipartitions of:

- the usual notion of vertical strips for partitions, see for instance [21, Chapter I].

- the usual notion of admissible boxes for multipartitions, see for instance [6], [7, Chapter 5].

Example 5.3 Let $\ell=3, e=4$ and

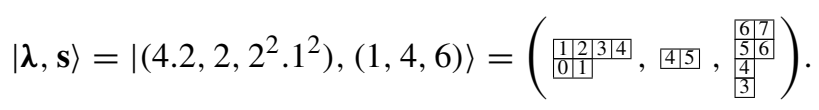


Then $\mathscr{V}(\boldsymbol{\lambda}, \mathbf{s})$ consists of

$X_{1}=((1,3,3),(2,3,3),(1,3,2),(1,5,1))$ with respective contents $8,7,6,5$, $X_{2}=((3,2,3),(4,2,3),(2,1,2),(2,3,1))$ with respective contents $5,4,3,2$, $X_{3}=((3,2,3),(4,2,3),(2,1,2),(3,1,2))$ with respective contents $5,4,3,2$, $X_{4}=((4,2,3),(2,1,2),(3,1,2),(4,1,2))$ with respective contents $4,3,2,1$, $X_{5}=((2,1,2),(3,1,2),(4,1,2),(5,1,2))$ with respective contents $3,2,1,0$, $X_{6}=((3,1,2),(4,1,2),(5,1,2),(3,1,1))$ with respective contents $2,1,0,-1$, $X_{7}=((3,1,2),(4,1,2),(5,1,2),(6,1,2))$ with respective contents $2,1,0,-1$, $X_{8}=((5,1,3),(4,1,2),(5,1,2),(3,1,1))$ with respective contents $2,1,0,-1$, $X_{9}=((5,1,3),(4,1,2),(5,1,2),(6,1,2))$ with respective contents $2,1,0,-1$, $X_{10}=((5,1,3),(6,1,3),(5,1,2),(3,1,1))$ with respective contents $2,1,0,-1$, $X_{11}=((5,1,3),(6,1,3),(5,1,2),(6,1,2))$ with respective contents $2,1,0,-1$, $X_{12}=((5,1,3),(6,1,3),(7,1,3),(3,1,1))$ with respective contents $2,1,0,-1$, $X_{13}=((5,1,3),(6,1,3),(7,1,3),(6,1,2))$ with respective contents $2,1,0,-1$, $X_{14}=((5,1,3),(6,1,3),(7,1,3),(8,1,3))$ with respective contents $2,1,0,-1$,

and so on.

\subsection{Action of the Heisenberg crystal operators $\tilde{b}_{\sigma}$}

We will define an order on the vertical $e$-strips of a given charged multipartition. First, let $\gamma=(a, b, j)$ and $\gamma^{\prime}=\left(a^{\prime}, b^{\prime}, j^{\prime}\right)$ be two boxes of $\mid \lambda$, s $\rangle$. Write $\gamma>\gamma^{\prime}$ if $\mathfrak{c}(\gamma)>\mathfrak{c}\left(\gamma^{\prime}\right)$ or $\mathfrak{c}(\gamma)=\mathfrak{c}\left(\gamma^{\prime}\right)$ and $j<j^{\prime}$.

Remark 5.4 Note that this is the total order used to define the good boxes in a charged $\ell$-partition, which characterises the Kashiwara crystals, see [7, Chapter 6].

By extension, let $>$ denote the lexicographic order induced by $>$ on $e$-tuples of boxes in a given charged $\ell$-partition. This restricts to a total order on $\mathscr{V}(\boldsymbol{\lambda}, \mathbf{s})$.

Definition 5.5 Let $|\lambda, \mathbf{s}\rangle$ be a charged $\ell$-partition. Denote simply $\mathscr{V}=\mathscr{V}(\lambda, \mathbf{s})$.

- The first good vertical $e$-strip of $|\lambda, \mathbf{s}\rangle$ is the maximal element $X_{1}$ of $\mathscr{V}$ with respect to $>$.

- Let $k \geq 2$. The $k$-th good vertical $e$-strip of $|\lambda, \mathbf{s}\rangle$ is the maximal element $X_{k}$ of

$$
\left\{X \in \mathscr{V} \mid X_{k-1}>X \text { and } X_{k-1} \cap X=\emptyset\right\}
$$

with respect to $>$.

Remark 5.6 This generalises the notion of good boxes for multipartitions, see again [6] or [7, Chapter 5].

In other terms, the greatest (with respect to $>$ ) vertical strip of $|\lambda, \mathbf{s}\rangle$ is good, and another admissible vertical strip is good except if one of its boxes already belongs to a previous good vertical strip. 
Example 5.7 We go back to Example 5.3. Then we have $X_{k}>X_{k-1}$ for all $k=$ $1, \ldots, 14$. Moreover, there are only four good addable vertical strips among these, namely $X_{1}, X_{2}, X_{6}$ and $X_{13}$.

For $\sigma=\left(\sigma_{1}, \sigma_{2}, \ldots\right) \in \Pi$, set

$$
\begin{aligned}
\tilde{c}_{\sigma}: B_{s} & \longrightarrow B_{s} \\
|\lambda, \mathbf{s}\rangle & \longmapsto|\boldsymbol{\mu}, \mathbf{s}\rangle
\end{aligned}
$$

where $\boldsymbol{\mu}$ is obtained from $\lambda$ by adding recursively $\sigma_{k}$ times the $k$-th good vertical $e$-strip for $k \geq 1$.

More explicitly, let $h$ be the number of non-zero parts of $\sigma$ and set recursively, for $k=1, \ldots, h$ and $m=0, \ldots, \sigma_{k}$

$$
\lambda^{(k, m)}= \begin{cases}\lambda & \text { if }(k, m)=(1,0) \\ \lambda^{\left(k-1, \sigma_{k-1}\right)} & \text { if } m=0 \text { and } k \geq 2 \\ \lambda^{(k, m-1)} \sqcup X_{k}\left(\lambda^{(k, m-1)}\right) & \text { otherwise, }\end{cases}
$$

where the $X_{k}(\lambda)$ denotes the $k$-th good vertical $e$-strip of $|\lambda, \mathbf{s}\rangle$. Then $\boldsymbol{\mu}=\lambda^{\left(h, \sigma_{h}\right)}$.

We are going to prove that the map $\tilde{c}_{\sigma}$ is well defined for all $\sigma \in \Pi$, and that it coincides with the Heisenberg crystal operator $\tilde{b}_{\sigma}$, see Theorem 5.11. .

Lemma 5.8 The map $\tilde{c}_{(1)}$ is well defined.

Proof Let $|\lambda, \mathbf{s}\rangle$ be a charged multipartition. We need to prove that the first good vertical strip $X$ of $|\lambda, \mathbf{s}\rangle$ is addable. Assuming it is not the case, then there exists a box $(a, b, j) \in X$ such that $(a-1, b, j) \notin X \sqcup \lambda$ and $(a-1, b-1, j) \in \lambda$. But $(a-1, b, j) \notin \lambda$ and $(a-1, b-1, j) \in \lambda$ implies $(a-1, b, j) \in X$, whence a contradiction.

Corollary 5.9 For all $\sigma \in \Pi$, the map $\tilde{c}_{\sigma}$ is well defined.

Proof If $\sigma=\emptyset$, then $\tilde{c}_{\sigma}=\mathrm{Id}$ and so is well defined. So we assume $\sigma \neq \emptyset$, say $\sigma=\left(\sigma_{1}, \sigma_{2}, \ldots\right)$. First of all, Lemma 5.8 and the recursive definition of $\tilde{c}_{\sigma}$ implies that $\tilde{c}_{(n)}$ is well defined for all $n \in \mathbb{Z}_{\geq 1}$ (so in particular for $n=\sigma_{1}$ ). We claim that for all $\lambda \in \Pi_{\ell}$ and for all $\ell$-charge $\mathbf{s}$, the second good vertical $e$-strip $X$ of $\tilde{c}_{\left(\sigma_{1}\right)}|\lambda, \mathbf{s}\rangle$ is addable $\sigma_{1}$ times recursively. In fact, this relies on the simple observation that $\tilde{c}_{\left(\sigma_{1}\right)}|\lambda, \mathbf{s}\rangle=\left|\lambda^{\left(1, \sigma_{1}\right)}, \mathbf{s}\right\rangle$ and that the second good vertical $e$-strip of $\lambda^{\left(1, \sigma_{i}\right)}$ is the second good vertical $e$-strip of $\lambda$ already. Since the first good vertical $e$-strip of $\lambda$ is addable (Lemma 5.8), it is straightforward from Definition 5.5 and the observation that the second good vertical $e$-strip of $\lambda^{\left(1, \sigma_{i}\right)}$ is addable. So the second good vertical $e$-strip of $\tilde{c}_{\left(\sigma_{1}\right)}|\lambda, \mathbf{s}\rangle$ is addable. But since $X_{2}\left(\lambda^{\left(1, \sigma_{1}\right)}\right)=X_{2}\left(\lambda^{(2,0)}\right)$ is addable, so is $X_{2}\left(\lambda^{(2, m)}\right)$ for all $m=1, \ldots, \sigma_{2}$ (since $\sigma_{2} \leq \sigma_{1}$ ), which proves the claim. Since $\sigma_{1} \geq \sigma_{2} \geq \sigma_{3} \geq \ldots$, the result follows by iterating up to $h$, the number of non-zero parts in $\sigma$. 
Example 5.10 Take again the values of Example 5.3. Then, for $\sigma=\left(2.1^{3}\right)$, we have

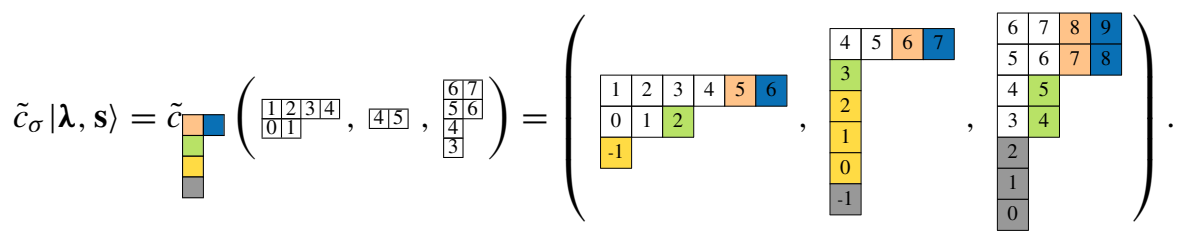

Each box of $\sigma$ corresponds to a vertical $e$-strip, the matching being given by the colors.

We will prove the following theorem.

Theorem 5.11 For all $\sigma \in \Pi$, we have

$$
\tilde{c}_{\sigma}=\tilde{b}_{\sigma}
$$

\subsection{Proof of Theorem 5.11}

The strategy for proving this result consists in starting from the doubly highest weight case, in which we know an explicit formula for $\tilde{b}_{\sigma}$. Then, we show that the $\tilde{c}_{ \pm \sigma}$ commute with the Kashiwara crystal operators for $\mathcal{U}_{p}^{\prime}\left(\widehat{\mathfrak{s l}_{\ell}}\right)$, then $\mathcal{U}_{q}^{\prime}\left(\widehat{\mathfrak{s l}_{e}}\right)$, and use the commutation of the Kashiwara crystals and the $\mathcal{H}$-crystal (see Definition 3.7) to conclude.

Proposition 5.12 Let $|\lambda, \mathbf{s}\rangle$ be a doubly highest weight vertex. Then, for all $\sigma \in \Pi$, we have

$$
\tilde{c}_{\sigma}|\lambda, \mathbf{s}\rangle=\tilde{b}_{\sigma}|\lambda, \mathbf{s}\rangle
$$

Proof We need to translate the explicit formula for $\tilde{b}_{\sigma}$, given in terms of abaci, in terms of $\ell$-partitions. By the correspondence given in Sect. 2.1, an $e$-period in the $\ell$-abacus $\mathcal{A}=\mathcal{A}(\boldsymbol{\lambda}, \mathbf{s})$ (see [12, Section 2.3]) corresponds to a good vertical $e$-strip in $|\lambda, \mathbf{s}\rangle$. Therefore, it yields an addable admissible vertical $e$-strip if $\left(j_{1}, d_{1}+1\right) \notin \mathcal{A}$ where $\left(j_{1}, d_{1}\right)$ is the first bead of the period. Thus, shifting the $k$-th $e$-period of $\mathcal{A}$ by $\sigma_{k}$ steps to the right amounts to adding the $k$-th good vertical strip of $|\lambda, \mathbf{s}\rangle$. In other terms $\tilde{b}_{\sigma}$ is the same as $\tilde{c}_{\sigma}$ when restricted to doubly highest weight vertices (cf. Definition 3.6).

Proposition 5.13 Let $|\lambda, \mathbf{s}\rangle$ be a highest weight vertexfor $\mathcal{U}_{q}^{\prime}\left(\widehat{\mathfrak{s l}_{e}}\right)$. Then, for all $\sigma \in \Pi$, we have

$$
\tilde{c}_{\sigma}|\lambda, \mathbf{s}\rangle=\tilde{b}_{\sigma}|\lambda, \mathbf{s}\rangle
$$

Proof Write $|\lambda, \mathbf{s}\rangle=\dot{F}_{\mathbf{j}}|\bar{\lambda}, \overline{\mathbf{s}}\rangle$ where $|\bar{\lambda}, \overline{\mathbf{s}}\rangle$ is the highest weight vertex for $\mathcal{U}_{p}^{\prime}\left(\widehat{\mathfrak{s l}_{\ell}}\right)$ associated to $|\lambda, \mathbf{s}\rangle$, and where $\dot{F}_{\mathbf{j}}=\tilde{\dot{f}}_{j_{r}} \ldots \tilde{\dot{f}}_{j_{1}}$ is a sequence of Kashiwara crystal operators of $\mathcal{U}_{p}^{\prime}\left(\widehat{\mathfrak{s l}_{\ell}}\right)$. Because of Theorem A.4, the two Kashiwara crystals commute, 
and thus $|\bar{\lambda}, \overline{\mathbf{s}}\rangle$ is a doubly highest weight vertex. We prove the result by induction on $r \in$ $\mathbb{N}$. If $r=0$, then $|\lambda, \mathbf{s}\rangle$ is a doubly highest weight vertex and this is Proposition 5.12. Suppose that the result holds for a fixed $r-1 \geq 0$. Write $|\boldsymbol{v}, \mathbf{t}\rangle=\tilde{\dot{f}}_{j_{r-1}} \ldots \tilde{\dot{f}}_{j_{1}}|\bar{\lambda}, \overline{\mathbf{s}}\rangle$, so that $|\lambda, \mathbf{s}\rangle=\tilde{\dot{f}}_{j_{r}}|\boldsymbol{v}, \mathbf{t}\rangle$. Because the crystal level-rank duality is realised in terms of abaci, we need to investigate one last time the action of $\tilde{\dot{f}}_{j_{r}}$ in the abacus. We know that $\tilde{\dot{f}}_{j_{r}}$ acts on an $e$-partition by adding its good addable $j_{r}$-box (i.e. of content $j_{r}$ modulo $\ell$ ). This corresponds to shifting a particular (white) bead one step up in the $e$-abacus of $|\boldsymbol{v}, \mathbf{s}\rangle$, see Example A.3 for an illustration. Now, if $j_{r} \neq 0$, then this corresponds to moving down a (black) bead in the $\ell$-abacus. Since the resulting abacus $\mathcal{A}(\boldsymbol{\lambda}, \mathbf{s})$ is again totally $e$-periodic (the two Kashiwara crystals commute), this preserves the $e$-period containing this bead. If $j_{r}=0$, then moving this white bead up corresponds to moving a black bead in position $(\ell, d)$ in the $\ell$-abacus (which is the first element of its $e$-period) down to position $(1, d-e)$. Again, this preserves the $e$-period. In both cases, the reduced $j_{r}$-word in the $e$-abacus (see [9, Section 4.2] for details) is unchanged and

$$
\tilde{\dot{f}}_{j_{r}} \tilde{c}_{\sigma}|\boldsymbol{v}, \mathbf{t}\rangle=\tilde{c}_{\sigma} \tilde{\dot{f}}_{j_{r}}|\boldsymbol{v}, \mathbf{t}\rangle
$$

Therefore, we have

$$
\begin{aligned}
\tilde{b}_{\sigma}|\lambda, \mathbf{s}\rangle & =\tilde{b}_{\sigma} \tilde{\dot{f}}_{j_{r}}|\boldsymbol{v}, \mathbf{t}\rangle \\
& =\tilde{\tilde{f}}_{j_{r}} \tilde{b}_{\sigma}|\boldsymbol{v}, \mathbf{t}\rangle \text { by Definition } 3.7 \\
& =\tilde{\tilde{f}}_{j_{r}} \tilde{c}_{\sigma}|\boldsymbol{v}, \mathbf{t}\rangle \text { by induction hypothesis } \\
& =\tilde{c}_{\sigma} \tilde{\dot{f}}_{j_{r}}|\boldsymbol{v}, \mathbf{t}\rangle \text { by }(*) \\
& =\tilde{c}_{\sigma}|\lambda, \mathbf{s}\rangle .
\end{aligned}
$$

We are now ready to prove Theorem 5.11. It remains to investigate the action of the Kashiwara crystal operators of $\mathcal{U}_{q}^{\prime}\left(\widehat{\mathfrak{s l}_{e}}\right)$.

Proof of Theorem 5.11 Write $|\lambda, \mathbf{s}\rangle=F_{\mathbf{i}}|\bar{\lambda}, \mathbf{s}\rangle$ where $|\bar{\lambda}, \mathbf{s}\rangle$ is the highest weight vertex for $\mathcal{U}_{q}^{\prime}\left(\widehat{\mathfrak{s l}_{e}}\right)$ associated to $|\lambda, \mathbf{s}\rangle$, and where $F_{\mathbf{i}}=\tilde{f}_{i_{r}} \ldots \tilde{f}_{i_{1}}$ is a sequence of Kashiwara crystal operators of $\mathcal{U}_{q}^{\prime}(\widehat{\mathfrak{s l}})$. We prove the result by induction on $r \in \mathbb{N}$. If $r=0$, then $|\lambda, \mathbf{s}\rangle$ is a highest weight vertex for $\mathcal{U}_{q}^{\prime}\left(\widehat{\mathfrak{s l}_{e}}\right)$ and this is Proposition 5.13. Suppose that the result holds for a fixed $r-1 \geq 0$. Write $|\boldsymbol{v}, \mathbf{s}\rangle=\tilde{f}_{i_{r-1}} \ldots \tilde{f}_{i_{1}} \mid \bar{\lambda}$, s $\rangle$, so that $|\lambda, \mathbf{s}\rangle=\tilde{f}_{i_{r}}|\boldsymbol{v}, \mathbf{s}\rangle$. Consider the reduced $i_{r}$-word for $|\boldsymbol{v}, \mathbf{s}\rangle$. Again, it is preserved by the action of $\tilde{c}_{\sigma}$ by Property (1) of Definition 5.1, and we have

$$
\tilde{\tilde{f}}_{j_{r}} \tilde{c}_{\sigma}|\boldsymbol{v}, \mathbf{t}\rangle=\tilde{c}_{\sigma} \tilde{\dot{f}}_{j_{r}}|\boldsymbol{v}, \mathbf{t}\rangle
$$

The commutation of $\tilde{b}_{\sigma}$ with $\tilde{f}_{i_{r}}$ together with the induction hypothesis completes the proof. 
Remark 5.14 Theorem 5.11 also yields an explicit description of the operators $\tilde{b}_{1, c}$, see their definition in Sect. 3.3. It acts on any charged $\ell$-partition by adding its $k$-th good vertical $e$-strip, where $c$ is the content of the $k$-th addable box of $\kappa$, where the boxes are ordered with respect to $>$ as defined in Sect. 5.2.

\subsection{Impact of conventions and relations with other results}

We end this section by mentioning an alternative realisation of the $\mathcal{H}$-crystal. In fact, some of the combinatorial procedures require some conventional choices, such as the maps $\tau$ and $\dot{\tau}$ yielding the level-rank duality, or the order on the boxes or on the vertical strips of multipartition. Like in the case of Kashiwara crystals, see e.g. [2, Remark 3.17] or [9, Remark 4.9] Choosing a different convention yields to a slightly different version of the Heisenberg crystal.

We have already seen in the proof of Theorem 4.4 that conventions can be adjusted to fit Losev's [19] or Iijima's [11] results about the action of a Heisenberg crystal operator or a Schur function respectively. This is done by using the conjugation of multipartitions. More precisely, one can decide to identify a charged $\ell$-partition $|\lambda, \mathbf{s}\rangle$ with $\left|\lambda^{\prime}, s\right\rangle$ instead of $|\lambda, s\rangle$ with the notations of Sect. 2.1.2. Then, the action of the Heisenberg crystal operators is expressed in terms of addable horizontal $e$-strips in the $\ell$-partition. This is equivalent to changing the order on the vertical strips, applying a Heisenberg crystal operator, and then conjugate.

One could also decide to exchange the role of $\tau$ and $\dot{\tau}$ in the level-rank duality, see "Appendix". Then, applying a Heisenberg crystal operator on an $\ell$-abacus would de decribed in terms of the corresponding $e$-abacus. More precisely, $\tilde{b}_{1, c}$ would then consists in shifting a $\ell$-period in the $e$-abacus in the particular case where it is totally $\ell$ periodic (see also the proof of Proposition 5.12). This permits to give an interpretation of Tingley's tightening procedure on descending $\ell$-abaci [26, Definition 3.8], thereby answering Question 1 of [26, Section 6].

Proposition 5.15 Let $\mathcal{A}$ be a descending $\ell$-abacus. For all $k \geq 1$, denote $T_{k}$ the $k$-th tightening operator associated to $\mathcal{A}$. We have

$$
T_{k}(\mathcal{A})=\tilde{b}_{-1, c}(\mathcal{A})
$$

where $c$ is determined by $k$.

Proof Recall that for this statement, we have considered the realisation of the Heisenberg crystal with the alternative version of level-rank-duality, swapping the roles of $\tau$ and $\dot{\tau}$, so that the operators $\tilde{b}_{-1, c}$ act on the $e$-abacus (rather than the $\ell$-abacus) by removing a vertical $\ell$-strip (Theorem 5.11). Remember that $\tilde{b}_{-1, c}=\tilde{b}_{\theta} \tilde{b}_{-\kappa}$ where $\theta$ depends on $c$. Now, if an $\ell$-abacus is descending [26, Definition 3.6], its corresponding $e$-abacus is totally $\ell$-periodic. In particular, the corresponding $e$-partition is a highest weight vertex in the $\mathcal{U}_{p}^{\prime}\left(\widehat{\mathfrak{s t}_{\ell}}\right)$-crystal, and we can use Proposition 5.13. In particular, $\tilde{b}_{-\kappa}$ and $\tilde{b}_{\theta}$ act by shifting $\ell$-periods in the $e$-abacus, and $\tilde{b}_{-1, c}$ shifts one $\ell$-period, say the $k$-th one, one step to the left in the $e$-abacus. This precisely what $T_{k}$ does. 
Finally, we mention that in another particular case, the Heisenberg crystal operator $\tilde{b}_{-\kappa}$ coincide with the canonical $\mathcal{U}_{q}^{\prime}\left(\widehat{\mathfrak{s l}}_{e}\right)$-isomorphism $\varphi$ (up to cyclage) of [8, Theorem 5.26] used to construct an affine Robinson-Schensted correspondence.

Proposition 5.16 Let $|\lambda, \mathbf{s}\rangle \in B_{s}$ be a doubly highest weight vertex. Write $\kappa=$ $\kappa(|\lambda, \mathbf{s}\rangle)$. We have

$$
\tilde{b}_{-\kappa} \xi^{h}|\lambda, \mathbf{s}\rangle=\varphi|\lambda, \mathbf{s}\rangle,
$$

where $h$ is the number of parts of $\kappa$ and $\xi$ is the cyclage isomorphism, see [8, Proposition 4.4].

Proof We use the notations of [8]. First of all, because of [9, Proposition 5.7], doubly highest weight vertices are cylindric in the sense of [8, Definition 2.3], and $\varphi|\lambda, \mathbf{s}\rangle$ is therefore well defined, and simply verifies $\varphi=\psi^{t}$ where $t$ is the number of pseudoperiods in $|\lambda, \mathbf{s}\rangle$ and $\psi$ is the reduction isomorphism. In fact, by definition of $\kappa$, we have $t=h$, the number of (non-zero) parts of $\kappa$, and it suffices to apply the cyclage $h$ times to $|\lambda, \mathbf{s}\rangle$ to match the formulas for $\tilde{b}_{-\kappa}$ and $\psi^{t}$.

\subsection{Examples of computations}

By Remark 5.14, the Heisenberg crystal can be computed recursively from its highest weight vertices, each of which yields a unique connected component, isomorphic to the Young graph by Proposition 3.11.

The empty multipartition is obviously a highest weight vertex for $\mathcal{H}$, and so is every multipartition with less than $e$ boxes. For instance, if $\ell=2, \mathbf{s}=(0,1)$ and $e=3$, we can compute the connected components of the Heisenberg crystal of $\mathcal{F}_{\mathbf{s}}$ with highest weight vertex (-, -) and (口, -). Up to rank 13, we get the following subgraph of the $\mathcal{H}$-crystal.

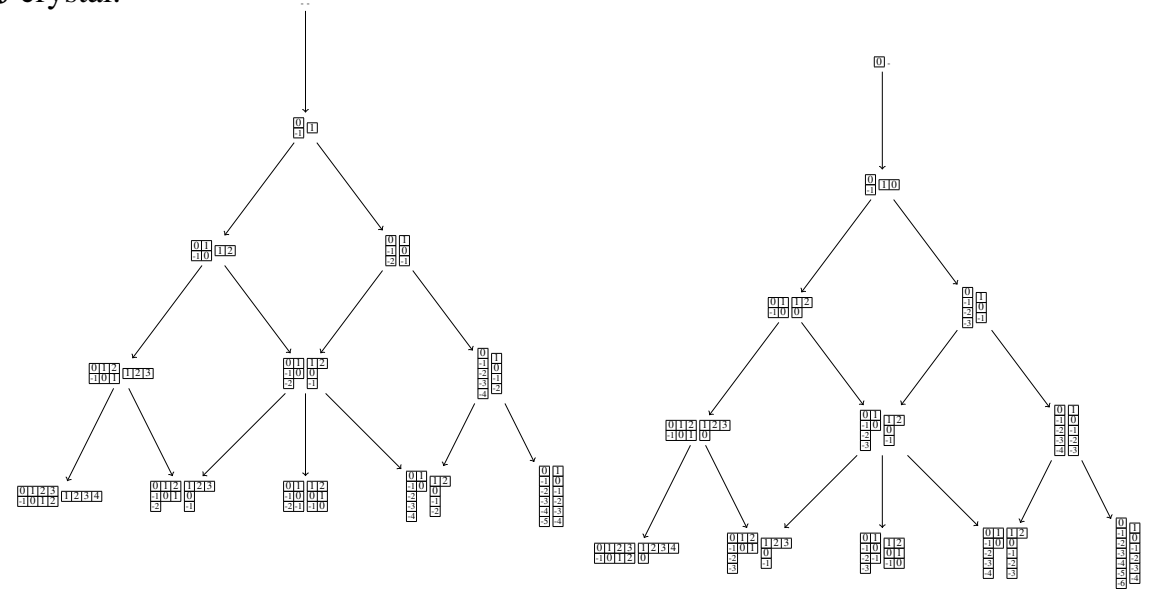

For $e=2$ and $\mathbf{s}=(3,2,5)$, the 3-partition $(2,1,4)$ is clearly a highest weight vertex for $\mathcal{H}$ by Theorem 5.11. The corresponding connected component, up to rank 17 is the following graph. 


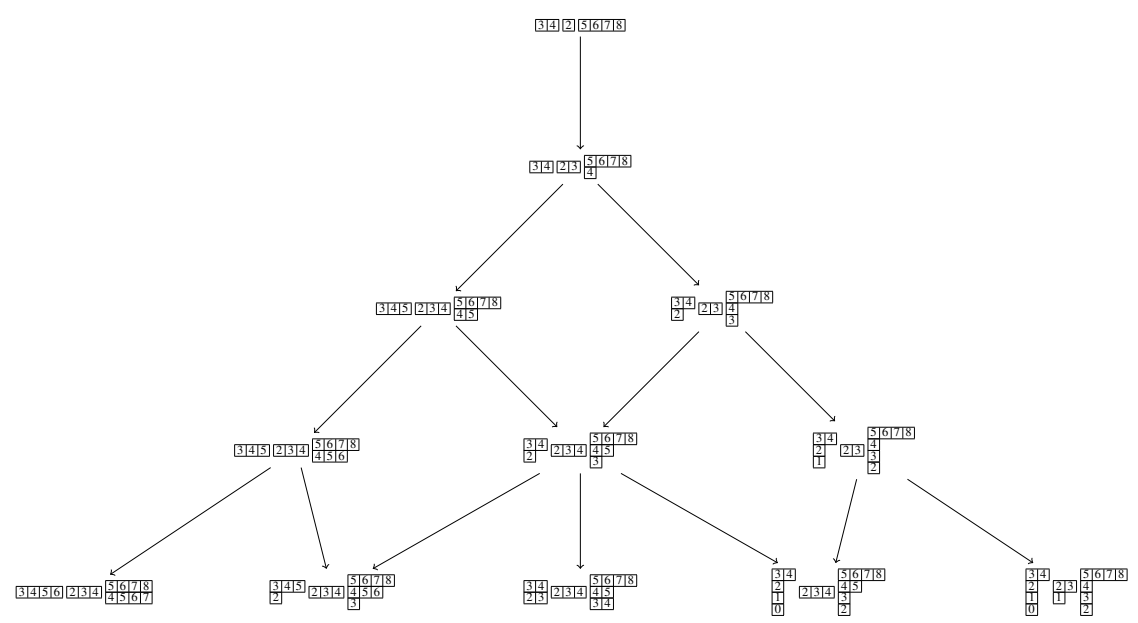

We also wish to give an example in the asymptotic case. Take $\ell=3, \mathbf{s}=(0,7,19)$, $\lambda=(1,3.2 .1,3.1)$ and $e=3$, so that $|\lambda, \mathbf{s}\rangle$ is a highest weight vertex for $\mathcal{H}$ and is 3 -asymptotic. We see in the following corresponding $\mathcal{H}$-crystal that the elements $\tilde{b}_{\sigma}$, for $|\sigma|<4$, only act on the third component of $|\lambda, \mathbf{s}\rangle$, but that $\tilde{b}_{\left(1^{4}\right)}$ acts already on the second component. This illustrates Theorem 4.4 (2).

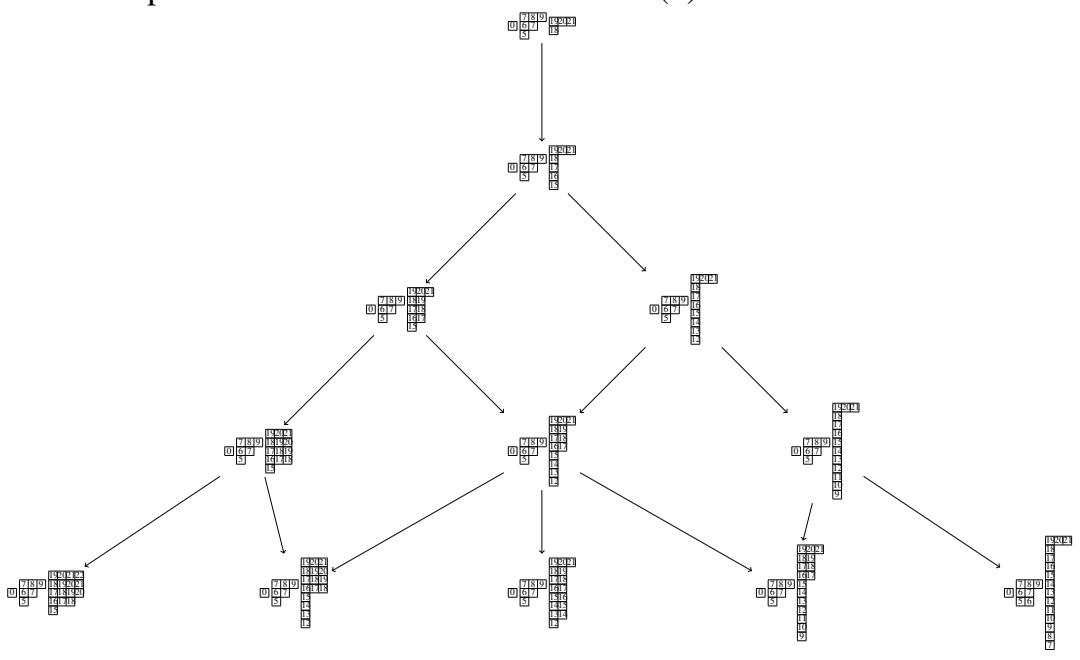

Acknowledgements Many thanks to Emily Norton for pointing out an inconsistency in the first version of this paper and for helpful conversations. 
Fig. 4 Relabelling bead positions in the $e$-abacus according to $\dot{\tau}^{-1}$, for $\ell=4$ and $e=3$. One should compare this with the labeling of Fig. 1

\begin{tabular}{|c|c|c|c|c|c|c|c|c|c|c|}
\hline & \multicolumn{6}{|c|}{$z=0$} & \multicolumn{4}{|c|}{$z=1$} \\
\hline & $\cdots$ & -11 & -8 & -5 & -2 & 1 & 4 & 7 & 10 & $\cdots$ \\
\hline & $\cdots$ & -10 & -7 & -4 & -1 & 2 & 5 & 8 & 11 & $\ldots$ \\
\hline & $\cdots$ & -9 & -6 & -3 & 0 & 3 & 6 & 9 & 12 & $\cdots$ \\
\hline & & -3 & -2 & -1 & 0 & 1 & 2 & 3 & 4 & \\
\hline
\end{tabular}

\section{Appendix A: Crystal level-rank duality}

We recall, using a slightly different presentation, the results of [9, Section 4] concerning the crystal version of level-rank duality.

There is a double affine quantum group action on the wedge space. In Sect. 2.2.1, we have explained how $\mathcal{U}_{q}^{\prime}\left(\widehat{\mathfrak{s l}_{e}}\right)$ acts on $\mathcal{F}_{s}$. It turns out that $\mathcal{U}_{p}^{\prime}\left(\widehat{\mathfrak{s l}_{\ell}}\right)$, where $p=-1 / q$, acts on $\mathcal{F}_{S}$ in a similar way. More precisely we will:

(1) index ordered wedges by charged $e$-partitions, using an alternative bijection $\dot{\tau}$,

(2) make $\mathcal{U}_{p}^{\prime}\left(\widehat{\mathfrak{s l}_{\ell}}\right)$ act on $\mathcal{F}_{s}$ via this new indexation by swapping the roles of $e$ and $\ell$ and replacing $q$ by $p$.

To define $\dot{\tau}$, recall that we have introduced the quantities $z(n) \in \mathbb{Z}, 1 \leq y(n) \leq \ell$ and $1 \leq x(n) \leq e$ for each $n \in \mathbb{Z}$. To each pair $(1, c) \in\{1\} \times \mathbb{Z}$, we associate the pair $\dot{\tau}(1, d)=(j, \bar{d}) \in\{1, \ldots, \ell\} \times \mathbb{Z}$ where

$$
j=x(-c) \text { and } d=-(y(-c)-1)+\ell z(-c) .
$$

In particular, $\dot{\tau}$ sends the bead in position $(1, c)$ into the rectangle $z(-c)$, on the row $x(-c)$ and column $y(-c)$ (numbered from right to left within each rectangle).

The map $\dot{\tau}$ is bijective and we can see $\dot{\tau}^{-1}$ as the following procedure:

(1) Divide the $\ell$-abacus into rectangles of size $e \times \ell$, where the $z$-th rectangle $(z \in \mathbb{Z})$ contains the positions $(j, d)$ for all $1 \leq j \leq \ell$ and $-e+1+z e \leq d \leq z e$.

(2) Relabel each $(j, d)$ by the second coordinate of $\dot{\tau}^{-1}(j, d)$, see Fig. 4 for an example.

(3) Replace the newly indexed beads on a 1-abacus according to this new labeling.

We see that $\dot{\tau}^{-1}$, so also $\dot{\tau}$, actually only depends on $e$, not on $\ell$. In fact, explicit formulas for $\dot{\tau}$ and $\dot{\tau}^{(1)}$ are given by

$$
\begin{aligned}
& \dot{\tau}(1, c)=\left(\left(\begin{array}{ll}
-c & \left.\bmod e)+1,-\left\lfloor\frac{-c}{e}\right\rfloor\right)
\end{array}\right.\right.
\end{aligned}
$$

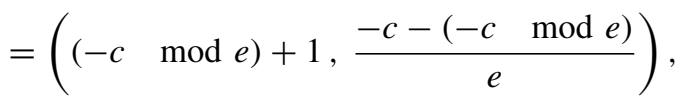

and

$$
\dot{\tau}^{-1}(j, d)=(1,-(j-1)+e d),
$$


and one notices that $\dot{\tau}$ corresponds to taking the usual $e$-quotient and the $e$-core of a partition, where the $e$-core is encoded in the $e$-charge. More precisely, the renumbering of the beads according to $\dot{\tau}$ is the well-know "folding" procedure used to compute the $e$-quotient, see [13].

Definition A.1 The (twisted) level-rank duality is the bijective map $\dot{\tau} \circ(.)^{\prime} \circ \tau^{-1}$

Remark A.2 The map $\dot{\tau} \circ \tau^{-1}$ already defines a level-rank duality, this was the one studied by Uglov [27]. However, the twisted version defined above (i.e. where the conjugation is added) is the one that is relevant when it comes to crystals, see Theorem A.4 below.

There is a convenient way to picture the crystal level-rank duality as follows. Starting from an $\ell$-abacus, stack copies on top of each other by translating by $e$ to the right. Then, extract a vertical slice of the resulting picture, and read off the corresponding $e$-partition by looking at the white beads (instead of black beads) in each column, starting from the leftmost one.

Example A.3 The abacus $\mathcal{A}(\lambda, \mathbf{s})$ with $\ell=4, e=3, \lambda=\left(1, \emptyset, 1^{3}, 5\right)$ and $\mathbf{s}=$ $(-1,-1,1,1)$ looks as follows (the origin is represented with the boldfaced vertical bar).

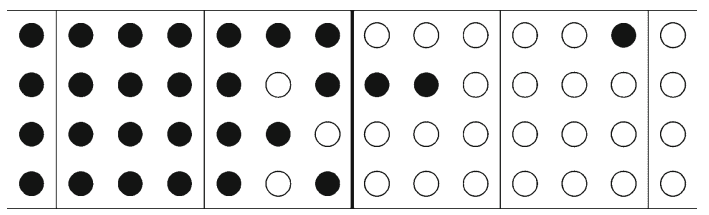

Stacking copies of $\mathcal{A}(\lambda, \mathbf{s})$ gives

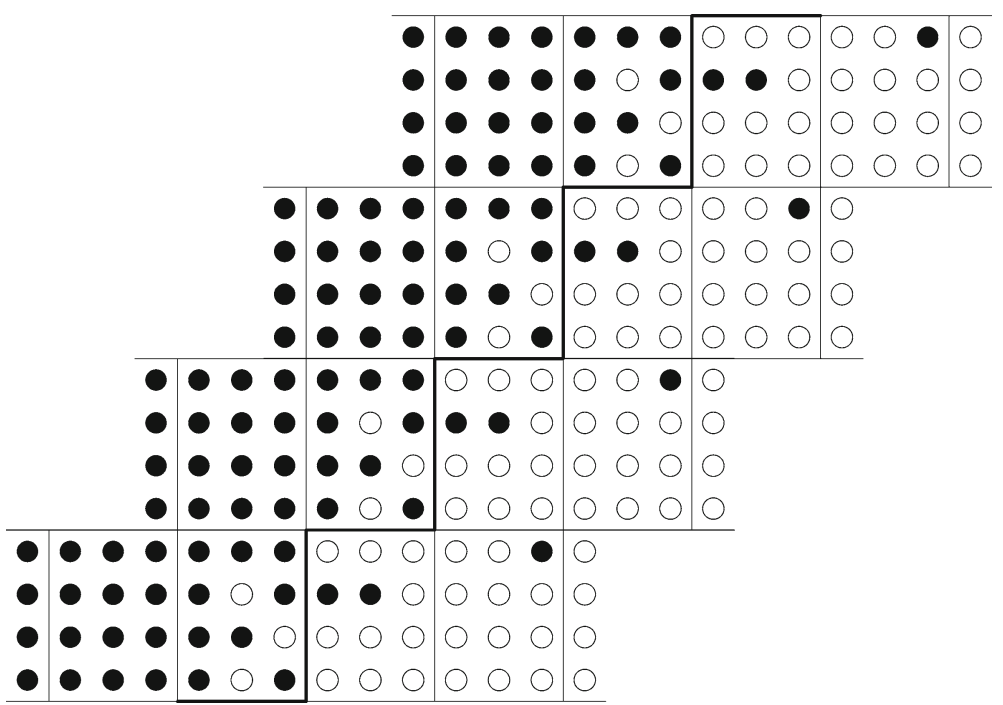

and extracting one vertical $e$-abacus yields 


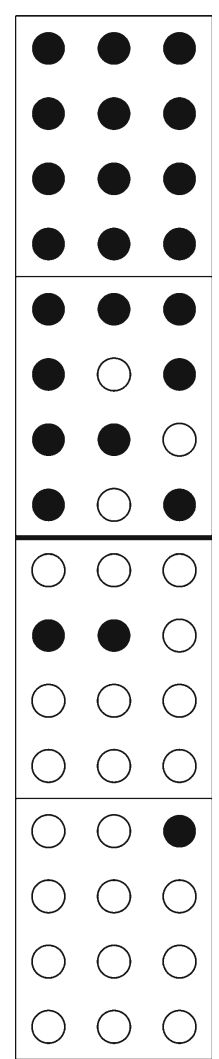

which corresponds to $\left|\left(1,2.1^{2}, 2.1^{4}\right),(-1,1,0)\right\rangle$.

We have the following induced maps

$$
\begin{gathered}
\bigoplus_{|\mathbf{s}|=s} \mathcal{F}_{\mathbf{s}} \stackrel{\tau^{-1}}{\rightleftharpoons \tau} \mathcal{F}_{s} \stackrel{{ }^{\prime}}{\rightleftharpoons} \mathcal{F}_{-s} \underset{\dot{\tau}^{-1}}{\stackrel{\dot{\tau}}{\rightleftharpoons}} \bigoplus_{|\dot{\mathbf{s}}|=s} \mathcal{F}_{\dot{\mathbf{s}}} \\
|\lambda, \mathbf{s}\rangle \longleftrightarrow|\lambda, s\rangle \longleftrightarrow\left|\lambda^{\prime},-s\right\rangle \longleftrightarrow|\dot{\lambda}, \dot{\mathbf{s}}\rangle
\end{gathered}
$$

Like $\tau$, the bijection $\dot{\tau}$ induces a $\mathcal{U}_{p}^{\prime}\left(\widehat{\mathfrak{s t}_{\ell}}\right)$-module isomorphism, and $\mathcal{F}_{s}$ has a $\mathcal{U}_{p}^{\prime}\left(\widehat{\mathfrak{s l}_{\ell}}\right)$-crystal, given by the same rule as the $\mathcal{U}_{q}^{\prime}\left(\widehat{\mathfrak{s l}_{e}}\right)$-crystal by swapping the roles of $e$ and $\ell$ and replacing $q$ by $p$.

\section{Theorem A.4 Via level-rank duality,}

(1) the $\mathcal{U}_{q}^{\prime}\left(\widehat{\mathfrak{s l}_{e}}\right)$-action and the $\mathcal{U}_{p}^{\prime}\left(\widehat{\mathfrak{s l}_{\ell}}\right)$-action on $\mathcal{F}_{s}$ commute, and

(2) the $\mathcal{U}_{q}^{\prime}\left(\widehat{\mathfrak{s l}_{e}}\right)$-crystal and the $\mathcal{U}_{p}^{\prime}\left(\widehat{\mathfrak{s l}_{\ell}}\right)$-crystal of $\mathcal{F}_{s}$ commute.

Proof The first point is essentially due to Uglov [27, Proposition 4.6], where he uses the non-twisted version of level-rank duality, see [9, Theorem 3.9] for the justification in the twisted case. The second point is [9, Theorem 4.8]. 


\section{References}

1. Ariki, S.: On the decomposition numbers of the Hecke algebra of $G(m, 1, n)$. J. Math. Kyoto Univ. 36(4), 789-808 (1996)

2. Brundan, J., Kleshchev, A.: Graded decomposition numbers for cyclotomic Hecke algebras. Adv. Math. 222, 1883-1942 (2009)

3. Dudas, O., Varagnolo, M., Vasserot, É.: Categorical actions on unipotent representations I. Finite unitary groups (2015). arXiv:1509.03269

4. Dudas, O., Varagnolo, M., Vasserot, É.: Categorical actions on unipotent representations of finite classical groups. In: Categorification and Higher Representation Theory. Contemporary Mathematics, vol. 683, pp. 41-104. American Mathematical Society, Providence, RI (2017)

5. Etingof, P.: Supports of irreducible spherical representations of rational Cherednik algebras of finite Coxeter groups. Adv. Math. 229, 2042-2054 (2012)

6. Foda, O., Leclerc, B., Okado, M., Thibon, J.-Y., Welsh, T.: Branching functions of $A_{n-1}^{(1)}$ and JantzenSeitz problem for Ariki-Koike algebras. Adv. Math. 141, 322-365 (1999)

7. Geck, M., Jacon, N.: Representations of Hecke Algebras at Roots of Unity. Springer, Berlin (2011)

8. Gerber, T.: Crystal isomorphisms in Fock spaces and Schensted correspondence in affine type A. Algebra Rep. Theory 18, 1009-1046 (2015)

9. Gerber, T.: Triple crystal action in Fock spaces. Adv. Math. (2016, to appear). https://doi.org/10.1016/ j.aim.2018.02.030

10. Gerber, T., Hiss, G., Jacon, N.: Harish-Chandra series in finite unitary groups and crystal graphs. Int. Math. Res. Not. 22, 12206-12250 (2015)

11. Iijima, K.: On a higher level extension of Leclerc-Thibon product theorem in $q$-deformed Fock spaces. J. Algebra 371, 105-131 (2012)

12. Jacon, N., Lecouvey, C.: A combinatorial decomposition of higher level Fock spaces. Osaka J. Math. 50(4), 897-920 (2013)

13. James, G., Kerber, A.: The Representation Theory of the Symmetric Group. Cambridge University Press, Cambridge (1984)

14. Jimbo, M., Misra, K.C., Miwa, T., Okado, M.: Combinatorics of representations of $U_{q}(\widehat{s l(n)})$ at $q=0$. Commun. Math. Phys. 136(3), 543-566 (1991)

15. Kashiwara, M.: Global crystal bases of quantum groups. Duke Math. J. 69, 455-485 (1993)

16. Kashiwara, M., Miwa, T., Stern, E.: Decomposition of $q$-deformed Fock spaces. Sel. Math. 1, 787-805 (1995)

17. Lascoux, A., Leclerc, B., Thibon, J.-Y.: Ribbon Tableaux, Hall-Littlewood Functions, quantum affine algebras and unipotent varieties. J. Math. Phys. 38, 1041-1068 (1997)

18. Leclerc, B., Thibon, J.-Y.: Littlewood-Richardson coefficients and Kazhdan-Lusztig polynomials. In: Combinatorial Methods in Representation Theory, volume 28 of Advanced Studies in Pure Mathematics. American Mathematical Society (2001)

19. Losev, I.: Supports of simple modules in cyclotomic Cherednik categories O (2015). arXiv:1509.00526

20. Lusztig, G.: Modular representations and quantum groups. Contemp. Math. 82, 58-77 (1989)

21. Macdonald, I.G.: Symmetric Functions and Hall Polynomials, 2nd edn. Oxford Mathematical Monographs, Oxford (1998)

22. Miwa, T., Jimbo, M., Date, E.: Solitons: Differential Equations, Symmetries and Infinite Dimensional Algebras. Cambridge University Press, Cambridge (2000)

23. Shan, P.: Crystals of Fock spaces and cyclotomic rational double affine Hecke algebras. Ann. Sci. Éc. Norm. Supér. 44, 147-182 (2011)

24. Shan, P., Vasserot, É.: Heisenberg algebras and rational double affine Hecke algebras. J. Am. Math. Soc. 25, 959-1031 (2012)

25. Stanley, R.P.: Enumerative Combinatorics, vol. 2. Cambridge University Press, Cambridge (2001)

26. Tingley, P.: Three combinatorial models for $\widehat{\mathfrak{s}_{n}}$ crystals, with applications to cylindric plane partitions. Int. Math. Res. Not. 143, 1-40 (2008)

27. Uglov, D.: Canonical bases of higher-level $q$-deformed Fock spaces and Kazhdan-Lusztig polynomials. Prog. Math. 191, 249-299 (1999)

28. Yvonne, X.: Bases canoniques d'espaces de Fock de niveau supérieur. Ph.D. thesis, Université de Caen (2005) 